Ann. Scient. Éc. Norm. Sup.,

$4^{\text {e }}$ série, t. 38, 2005, p. 116 à 153.

\title{
EXPANDING MAPS ON CANTOR SETS AND ANALYTIC CONTINUATION OF ZETA FUNCTIONS
}

\author{
BY FRÉDÉRIC NAUD
}

\begin{abstract}
In this paper, we study a class of Ruelle dynamical zeta functions related to uniformly expanding maps on Cantor sets. We show that under a non-local integrability condition, the zeta function enjoys a non-vanishing analytic continuation in a strip on the left of the line of absolute convergence. Applying these results to Fuchsian Schottky groups and Julia sets yields precise asymptotics of the number of closed geodesics for convex co-compact surfaces and the distribution of periodic points for a family of Cantor-like Julia sets.
\end{abstract}

(C) 2005 Elsevier SAS

RÉSUMÉ. - Dans cet article, on s'intéresse à une classe de fonctions zêta de Ruelle associées aux applications markoviennes uniformément dilatantes générant des ensembles de Cantor. On montre, sous une hypothèse de non intégrabilité locale, que ces fonctions zêta admettent un prolongement analytique sans zéros dans une bande à gauche de l'axe de convergence absolue. Appliqué aux ensembles limites de groupes de Schottky fuchsiens, ce résultat implique une asymptotique précise de la fonction de comptage des géodésiques périodiques sur les surfaces convexes co-compactes. On donne également un exemple d'application à des résultats de comptage pour une famille d'ensembles de Julia quadratiques de type Cantor.

(C) 2005 Elsevier SAS

\section{Introduction and statement of results}

The first prime orbit theorem for the geodesic flow on compact Riemann surfaces dates back to the work of Huber [18] and was later improved by Hejhal [17] and Randol [35]. Their result is, in a nutshell, the following.

THEOREM 1.1. - Let $M$ be a compact Riemann surface of constant curvature -1, and by $N(T)$ we denote the number of primitive closed geodesics $\gamma$ with length $l(\gamma) \leqslant T$. Then there exists $0<\alpha<1$ such that as $T \rightarrow \infty$,

$$
N(T)=\operatorname{li}\left(\mathrm{e}^{T}\right)+\mathrm{O}\left(\mathrm{e}^{\alpha T}\right)
$$

where $\operatorname{li}(x)=\int_{2}^{x} \frac{d t}{\log (t)}$.

We point out that $\alpha$ is in fact explicit and is related to the low eigenvalues of the Laplace Beltrami operator on $M$. Similar asymptotics for non-compact Riemann surfaces of finite volume were proved by Sarnak [39] (see also the book by Iwaniec [19]). The proofs of the above theorem are based on the celebrated Selberg trace formula [40] or the Selberg zeta function defined for 
$\operatorname{Re}(s)>1$ by the infinite product

$$
Z_{M}(s)=\prod_{k=0}^{+\infty} \prod_{\gamma \in \mathcal{P}}\left(1-\mathrm{e}^{-(s+k) l(\gamma)}\right),
$$

where $\mathcal{P}$ denotes the set of primitive closed geodesics. The key argument is the precise knowledge of the non-trivial zeros of $Z_{M}(s)$ which are in one-to-one correspondence with the point spectrum of the Laplace-Beltrami operator (see [17,19]).

In the case of non-compact Riemann surfaces of infinite volume with finite geometry, even if an exact trace formula is still valid [14], much less is already known. Indeed, the point spectrum of the Laplace operator can be empty, and the relevant spectral quantities are the resonances (the scattering spectrum). Resonances can be defined as poles of the meromorphic continuation (see [23]) to the half-plane $\{\operatorname{Re}(s) \leqslant 1 / 2\}$ of the resolvent of the Laplacian $\Delta$

$$
R(s): C_{0}^{\infty}(M) \rightarrow C^{\infty}(M),
$$

where $R(s)=(\Delta-s(1-s))^{-1}$. The lack of symmetry and our poor knowledge of the scattering spectrum are the main difficulties encountered when trying to extend the finite area results.

If $M$ is convex co-compact (i.e. of finite geometry with no cusps), then the following was proved conditionally by Guillopé [15] using estimates on heat kernels and later unconditionally by Lalley [21] using symbolic dynamics and renewal equations techniques.

THEOREM 1.2. - Let $M$ be a convex co-compact surface of constant negative curvature -1, and let $\delta$ be the topological entropy of the geodesic flow on the unit tangent bundle SM. Then as $T \rightarrow+\infty$,

$$
N(T) \sim \frac{\mathrm{e}^{\delta T}}{\delta T} .
$$

If we view $M$ as a quotient $\Gamma \backslash \mathbb{H}^{2}$ of the hyperbolic plane $\mathbb{H}^{2}$ by a Fuchsian group $\Gamma, 0<\delta<1$ is exactly the dimension of the limit set of $\Gamma$. The proof of Lalley uses ergodic theory and transfer operator techniques and can also be derived from the work of Parry and Pollicott [28] on zeta functions.

In the case of convex co-compact surfaces, the Selberg zeta function $Z_{M}(s)$ is known to be an entire function [16], whose non-trivial zeros are given by the resonances and the finite point spectrum. The Selberg zeta function $Z_{M}(s)$ is non-vanishing for $\operatorname{Re}(s)>\delta$ and as a consequence of the weak-mixing property of the geodesic flow and the theory in [28], does not vanish on the line $\{\operatorname{Re}(s)=\delta\}$ except for $s=\delta$ which is a simple zero. Our main result is the following.

THEOREM 1.3. - Let $M$ be a convex co-compact surface of constant negative curvature -1. There exists $\varepsilon>0$ such that $Z_{M}(s)$ is analytic and non-vanishing on the set $\{\operatorname{Re}(s)>\delta-\varepsilon\}$, except at $s=\delta$ which is a simple zero.

Notice that this result is obvious if $\delta>\frac{1}{2}$ since by [29] the only possible zeros of $Z_{M}(s)$ in the half-plane $\left\{\operatorname{Re}(s)>\frac{1}{2}\right\}$ are real and related to the (finite) point spectrum of $\Delta$ in $\left(0, \frac{1}{4}\right)$. If $\delta \leqslant \frac{1}{2}$, then this result is non-trivial and has deep consequences on the resonances.

Using Theorem 1.3 and the upper bound on the growth of $Z_{M}(s)$ proved in [16] (or alternatively the upper bound of Theorem 1.7) we get the following improvement of Theorem 1.2.

THEOREM 1.4. - Let $M$ be a convex co-compact surface of constant negative curvature -1. Let $N(T)$ be the number of primitive closed geodesics of length less than T. Then there exists 
$0<\alpha<\delta$, such that as $T \rightarrow+\infty$,

$$
N(T)=\operatorname{li}\left(\mathrm{e}^{\delta T}\right)+\mathrm{O}\left(\mathrm{e}^{\alpha T}\right) .
$$

A standard way to prove Theorem 1.4 is to follow the number theoretic arguments of Pollicott and Sharp [34]. An alternative way to derive Theorem 1.4 from Theorem 1.3 is to use the wave trace formula, see [26] where we actually give an explicit expression for $\alpha$.

Another example of zeta functions related to Cantor sets is provided by hyperbolic Julia sets. Consider the quadratic family $f(z)=z^{2}+c$, with $c<-2$. The Julia set $J$ is hyperbolic and is a Cantor set on the real line. We will denote by $\delta$ the Hausdorff dimension of $J$. Using suitable spaces of analytic functions, the zeta function defined for $\operatorname{Re}(s)>\delta$ by

$$
Z(s)=\exp \left(-\sum_{n=1}^{+\infty} \frac{1}{n} \sum_{f^{n} z=z} \frac{\left|\left(f^{n}\right)^{\prime}(z)\right|^{-s}}{1-\left|\left(f^{n}\right)^{\prime}(z)\right|^{-1}}\right)
$$

can be extended (see the paper of Strain and Zworski [42]) to an entire function. We will show the following.

THEOREM 1.5. - There exists $\varepsilon>0$ such that $Z(s)$ has no zeros in the half-plane $\{\operatorname{Re}(s)>\delta-\varepsilon\}$, except at $s=\delta$ which is a simple zero.

Let $\mathcal{P} e r$ denote the set of primitive periodic orbits of $f: J \rightarrow J$. Given

$$
\hat{x}=\left\{x, f x, \ldots, f^{n-1} x\right\} \in \mathcal{P} e r,
$$

with $f^{n} x=x$, we define the multiplier $\lambda(\hat{x})$ by $\lambda(\hat{x})=\left|\left(f^{n}\right)^{\prime}(x)\right|$. Following the arguments of [34], Theorem 1.5 implies the next counting result.

THEOREM 1.6. - There exists $0<\alpha<\delta$ such that as $X \rightarrow+\infty$,

$$
\#\{\hat{x} \in \mathcal{P} \text { er: } \lambda(\hat{x}) \leqslant X\}=\operatorname{li}\left(X^{\delta}\right)+\mathrm{O}\left(X^{\alpha}\right) .
$$

We conjecture that this kind of growth law for the multipliers should hold for generic ${ }^{1}$ rational hyperbolic Julia sets. In the spirit of Section 3, it would be especially interesting to look at families of hyperbolic Blashke products, whose dynamics are very similar to Fuchsian groups.

Theorems 1.3 and 1.5 are in fact consequences of a much more general result that can be stated in the set up of Ruelle dynamical zeta functions related to regular Cantor sets.

Let $\left(I_{i}\right)_{1 \leqslant i \leqslant k}$ be $k \geqslant 2$ closed, disjoint and bounded intervals included in $\mathbb{R}$. Let

$$
T: I=\bigcup_{i=1}^{k} I_{i} \rightarrow \mathbb{R}
$$

be a map such that $T_{i}:=\left.T\right|_{I_{i}}$ is $C^{2}$ on each ${ }^{2} I_{i}$. We assume that $T$ has in addition the following properties.

\footnotetext{
${ }^{1}$ This asymptotic certainly does not hold for $c=0$, where $Z(s)=1-2^{1-s}$ but we think that the conclusion of Theorem 1.5 is true for $c \neq 0$ in the main cardioid of the Mandelbrot set. It is likely that "generic" should be understood in the measure theoretic sense.

${ }^{2}$ We assume this smoothness for simplicity but in fact, $C^{1+\varepsilon}$ is enough.

$4^{\mathrm{e}}$ SÉRIE - TOME $38-2005-\mathrm{N}^{\circ} 1$
} 
1. (Eventually expanding) There exist $\gamma>1, D>0$ such that for all $N \geqslant 1$ and all $x \in T^{-N+1} I$, we have

$$
\left|\left(T^{N}\right)^{\prime}(x)\right| \geqslant D^{-1} \gamma^{N}
$$

2. (Markov property) For all $i, j, T\left(I_{j}\right) \cap \operatorname{Int}\left(I_{i}\right) \neq \emptyset \Rightarrow T\left(I_{j}\right) \supset I_{i}$.

We define a $k \times k$ transition matrix $A$ in the usual way by setting

$$
A(i, j)= \begin{cases}1 & \text { if } T\left(I_{i}\right) \supset I_{j}, \\ 0 & \text { otherwise. }\end{cases}
$$

We assume in the following that $T$ is topologically mixing on the non-wandering set, that is $A$ is irreducible aperiodic i.e. there exists a power $p_{0}>0$ such that $A^{p_{0}}>0$. We can associate a subshift of finite type to the transition matrix $A$

$$
\Sigma_{A}^{+}=\left\{\left(x_{n}\right)_{n \in \mathbb{N}} \in\{1, \ldots, k\}^{\mathbb{N}}: \forall i \geqslant 0, A\left(x_{i}, x_{i+1}\right)=1\right\} .
$$

The shift map is defined as usual by $(\sigma x)_{n}=x_{n+1}$ for all $n \geqslant 0$. Under the above assumptions, the non-wandering set $K=\bigcap_{i=0}^{\infty} T^{-i}(I)$ is called a $T$-invariant regular Cantor set, and the dynamical system $(K, T)$ is topologically conjugated to the subshift of finite type $\left(\Sigma_{A}^{+}, \sigma\right)$. This conjugacy is done via the map $\Pi: \Sigma_{A}^{+} \rightarrow K$, where

$$
\Pi(x)=\bigcap_{i \geqslant 0} T^{-i}\left(I_{x_{i}}\right) .
$$

Because of the uniform hyperbolicity of $T, \Pi$ is Lipschitz on $\Sigma_{A}^{+}$with respect to a well chosen standard ultrametric (see again [28]).

The functional space we will use throughout this paper is the Banach space $C^{1}(I)$ of complexvalued, $C^{1}$ functions on $I=\bigcup_{i=1}^{k} I_{i}$, endowed with the norm $\|f\|_{C^{1}}=\|f\|_{\infty}+\left\|f^{\prime}\right\|_{\infty}$. In the following $\tau \in C^{1}(I)$ is a real-valued, eventually positive function, that is there exists $N \geqslant 1$ such that the sum $\tau^{N}(x)$ defined by $\tau^{N}(x)=\tau(x)+\tau(T x)+\cdots+\tau\left(T^{N-1} x\right)$ is strictly positive for all $x \in T^{-N}(I)$.

Because $\tau$ is eventually positive, the variational principle implies that the pressure function (see the classical monographs $[28,7,45]$ for different equivalent definitions of the topological pressure)

$$
P(-x \tau)=\sup _{\mu \in \mathcal{M}_{\mathrm{inv}}}\left(h_{\mu}(T)-x \int_{K} \tau d \mu\right)
$$

$\left(\mathcal{M}_{\text {inv }}\right.$ denotes the set of $T$-invariant probability measures and $h_{\mu}(T)$ the measure-theoretic entropy) is strictly decreasing and has a unique positive zero denoted by $s_{0}$.

Let $\zeta(s)$ be the dynamical zeta function defined by the generalized Fredholm determinant

$$
\zeta(s)=\exp \left(\sum_{n=1}^{+\infty} \frac{1}{n} \sum_{T^{n} x=x} \mathrm{e}^{-s \tau^{n}(x)}\right),
$$

which is analytic (see [28]) for $\operatorname{Re}(s)>s_{0}$, where $P\left(-s_{0} \tau\right)=0$. Under a non-integrability condition (NLI), to be defined precisely in the next section, we have the following.

THEOREM 1.7. - Assume that $\tau \in C^{1}(I)$ has the property (NLI). Then for all $\epsilon>0$, there exists $\varepsilon_{0}=\varepsilon_{0}(\epsilon)>0$ such that $\zeta(s)$ has an analytic extension without zeros to $\operatorname{Re}(s)>s_{0}-\varepsilon_{0}$ 
except at $s=s_{0}$ which is a simple pole with residue 1 . Moreover, we have for all $s_{0} \geqslant \operatorname{Re}(s)>$ $s_{0}-\varepsilon_{0}$ and $|\operatorname{Im}(s)|$ large, the estimate

$$
\exp \left(-C_{\epsilon}|\operatorname{Im}(s)|^{2+\epsilon}\right) \leqslant|\zeta(s)| \leqslant \exp \left(C_{\epsilon}|\operatorname{Im}(s)|^{2+\epsilon}\right),
$$

for a well chosen constant $C_{\epsilon}>0$.

This paper is organized as follows. In the next section, we give a precise definition of the non local integrability condition (NLI) and show how Theorem 1.7 can be deduced from a spectral estimate (Theorem 2.3) of an analytic family of "twisted" transfer operators. In Section 3 , motivated by the paper of Strain and Zworski [42], we focus on the family of Julia sets described above and give a proof of Theorem 1.5. In Section 4, we recall the structure of Fuchsian Schottky groups which are used to uniformize convex co-compact surfaces. The action of Schottky groups on the boundary $\partial \mathbb{H}^{2}$ of the hyperbolic plane induces an expanding map on the limit set (the Bowen-Series map) whose periodic points are related to the closed geodesics of the corresponding surface. The Ruelle zeta function of this map is therefore closely related to the Selberg zeta function. To apply Theorem 1.7 to convex co-compact surfaces, we need to prove condition (NLI) and this is done in Section 4.2. The proof of the Theorem 2.3 occupies Section 5 and is based on the techniques of Dolgopyat [11] which have to be significantly modified for our purpose. Indeed, the disconnected structure of the Cantor set adds some technical difficulties like the existence of triadic partitions (see Section 7), or the Federer property of equilibrium measures (see Section 6) which are non-trivial in our case. We point out that since the original work of Dolgopyat on decay of correlations for Anosov flows [11], his techniques have been extended to cover a wide range of problems including billiard flows [41], counting problems on surfaces of negative curvature [33,2] and more recently to the analysis of Euclidian algorithms [5].

Due to the generality of Theorem 1.7, it is likely that it could be applied to counting problems on convex co-compact surfaces of variable negative curvature and various zeta functions related to Cantor sets. We also expect that these techniques can be extended to higher dimensional Schottky manifolds and zeta functions related to more general Kleinian groups.

\section{Non-integrability and contraction of transfer operators}

We use the same notations as in Section 1. The complex transfer operator $\mathcal{L}_{s}$ is defined for all $s \in \mathbb{C}$ by

$$
\mathcal{L}_{s}(f)(x)=\sum_{T y=x} \mathrm{e}^{-s \tau(y)} f(y)
$$

and acts as a bounded linear operator on $C^{1}(I)$.

We recall the definition of the so-called "temporal distance" function which is involved in recent results of ergodic theory $[11,41]$, and plays a crucial role in the proof. Given an eventually positive function $\tau \in C^{1}(I)$, we define the function $\Delta_{\xi}(u, v)$ for all $\xi \in \Sigma_{A}^{-}$and $u, v \in I_{j}$ with $T\left(I_{\xi_{0}}\right) \supset I_{j}$ by

$$
\Delta_{\xi}(u, v)=\sum_{j=0}^{+\infty} \tau\left(T_{\xi_{-j}}^{-1} \circ \cdots \circ T_{\xi_{0}}^{-1} u\right)-\tau\left(T_{\xi_{-j}}^{-1} \circ \cdots \circ T_{\xi_{0}}^{-1} v\right) .
$$

We recall that $\Sigma_{A}^{-}$is the space of negative sequences $\left(x_{i}\right)_{i \leqslant 0}$ with $A\left(x_{i}, x_{i+1}\right)=1$ for all $i \leqslant-1$. Because of the uniformly contracting properties of the inverse branches $T_{\xi_{i}}^{-1}, \Delta_{\xi}(u, v)$ is a $C^{1}$

$4^{\mathrm{e}}$ SÉRIE - TOME $38-2005-\mathrm{N}^{\circ} 1$ 
function on $I_{j} \times I_{j}$. Given $j \in\{1, \ldots, k\}, \eta, \xi \in \Sigma_{A}^{-}$with $T\left(I_{\xi_{0}}\right) \supset I_{j}$ and $T\left(I_{\eta_{0}}\right) \supset I_{j}$, the temporal distance function $\varphi_{\xi, \eta}(u, v)$ is well defined for all $u, v \in I_{j}$ by

$$
\varphi_{\xi, \eta}(u, v)=\Delta_{\xi}(u, v)-\Delta_{\eta}(u, v) .
$$

The definition we give here is the same as in [13], but in a smoother setup. Indeed, the definition of the temporal distance function of [13] concerns a symbolic setup where the corresponding function is only Hölder.

DEFINITION 2.1. - An eventually positive function $\tau \in C^{1}(I)$ has the non-local integrability property (NLI) if there exist $j \in\{1, \ldots, k\}, \xi, \eta \in \Sigma_{A}^{-}$with $T\left(I_{\xi_{0}}\right) \cap T\left(I_{\eta_{0}}\right) \supset I_{j}$ and $u_{0}, v_{0} \in$ $K \cap I_{j}$ such that

$$
\frac{\partial \varphi_{\xi, \eta}}{\partial u}\left(u_{0}, v_{0}\right) \neq 0 .
$$

If $T$ and $\tau$ are real analytic then it turns out that (NLI) is equivalent to the fact that $\varphi_{\xi, \eta}(u, v)$ is non-identically vanishing (see our two examples) and this is exactly the definition of nonlocal integrability given by Dolgopyat in a symbolic setting (see the appendix in [12]). This terminology "non-integrable" goes back to Anosov [3] where he showed that if the temporal distance function is identically vanishing on a section of an Anosov flow, then the strong stable and the strong unstable distributions of the flow are jointly integrable.

We also need to recall the definition of a non-lattice suspension taken from [28].

Definition 2.2. - The function $\tau \in C^{1}(I)$ is called non-lattice if there is no function $L: K \rightarrow m \mathbb{Z}$ with $m>0$ and $f: K \rightarrow \mathbb{R}, f$ Lipschitz on $K$, such that for all $x \in K$,

$$
\tau(x)=f(x)-f \circ T(x)+L(x) .
$$

The relation $\tau(x)=f(x)-f \circ T(x)+L(x)$ is often called in ergodic theory a "cohomological equation", and we will use this terminology in the next sections. If $\tau$ is non-lattice, then by [28], p. 96, Theorem 6.3, $\zeta(s)$ has an non-vanishing analytic extension to the half-plane $\left\{\operatorname{Re}(s)>s_{0}\right\}$ except at $s=s_{0}$ which is a simple pole with residue 1 . We recall that $s_{0}>0$ is defined by $P\left(-s_{0} \tau\right)=0$. We can now state the central result of this paper.

THEOREM 2.3. - Assume that an eventually positive function $\tau \in C^{1}(I)$ has the property $(N L I)$. Then for all $\epsilon>0$, there exist $C_{\epsilon}>0, \varepsilon_{0}(\epsilon)>0, t_{0}>0,0<\rho_{\epsilon}<1$ such that for all $s_{0}-\varepsilon_{0}<\operatorname{Re} s \leqslant s_{0}$ and $|\operatorname{Im} s| \geqslant t_{0}$,

$$
\left\|\mathcal{L}_{s}^{n}\right\|_{C^{1}} \leqslant C_{\epsilon}|\operatorname{Im} s|^{1+\epsilon} \rho_{\epsilon}^{n},
$$

where $s_{0}$ is the unique real number such that $P\left(-s_{0} \tau\right)=0$ and $P$ denotes the topological pressure on $K$.

The condition (NLI) implies the non-lattice (see the proof below). There exist examples of locally integrable flows which are non-lattice and cannot satisfy the conclusions of this theorem $[30,37]$. The definition of the non-integrability given in [13] is stronger than ours and difficult to check in the examples because of the lack of differentiability on symbolic spaces. On the other hand, the proof of Theorem 2.3 becomes more complicated than its analogue in [13], mainly because the measure theoretic and metric structures of $K$ are not so naturally related as in the symbolic setting.

The transfer operator estimate of Theorem 2.3 implies Theorem 1.7. 
Proof. - For all $s \in \mathbb{C}$ and $n \geqslant 1$, let $Z_{n}(s)$ be the weighted sum on the periodic points

$$
Z_{n}(s)=\sum_{T^{n} x=x} \mathrm{e}^{-s \tau^{n}(x)} .
$$

Let $\chi_{i} \in C^{1}(I)$ be the characteristic function of the interval $I_{i}$. Using some ideas of Ruelle [38], one can show the following estimate (see [33]).

PROPOSITION 2.4. - There exist $x_{1}, \ldots, x_{k} \in I_{1}, \ldots, I_{k}$ such that for all $\alpha>0$, there exists $a$ constant $C_{\alpha}>0$ such that

$$
\left|Z_{n}(s)-\sum_{i=1}^{k} \mathcal{L}_{s}^{n}\left(\chi_{i}\right)\left(x_{i}\right)\right| \leqslant C_{\alpha}|\operatorname{Im}(s)| \sum_{m=2}^{n}\left\|\mathcal{L}_{s}^{n-m}\right\|_{C^{1}}\left(\frac{1}{\gamma} \mathrm{e}^{\alpha+P(-\operatorname{Re}(s) \tau)}\right)^{m},
$$

for all $|\operatorname{Im}(s)|$ large enough and all $\operatorname{Re}(s)$ in a bounded set.

The detailed proof of this result is postponed to Appendix A of this paper. We now fix $\epsilon>0$ and $C=C_{\epsilon}>0$ as in Theorem 2.3. Taking $\operatorname{Re}(s)$ close enough to $s_{0}$ and applying Theorem 2.3, we get for all $|\operatorname{Im}(s)|$ large enough

$$
\begin{aligned}
\left|Z_{n}(s)\right| & \leqslant\left|Z_{n}(s)-\sum_{i=1}^{k} \mathcal{L}_{s}^{n}\left(\chi_{i}\right)\left(x_{i}\right)\right|+C k|\operatorname{Im}(s)|^{1+\epsilon} \rho_{\epsilon}^{n} \\
& \leqslant C k|\operatorname{Im}(s)|^{1+\epsilon} \rho_{\epsilon}^{n}+C C_{\alpha}|\operatorname{Im}(s)|^{2+\epsilon} \rho_{\epsilon}^{n} \sum_{m=2}^{n}\left(\frac{1}{\rho_{\epsilon} \gamma} \mathrm{e}^{\alpha+P(-\operatorname{Re}(s) \tau)}\right)^{m} .
\end{aligned}
$$

We remark now that it is always possible to choose $1>\rho_{\epsilon}>\frac{1}{\gamma}$ (we recall that $\gamma$ is the expanding rate of the map $T)$. Since $P\left(-s_{0} \tau\right)=0$, by continuity of the topological pressure and by taking $\alpha$ small enough, for all $\operatorname{Re}(s)$ close to $s_{0}$ we get

$$
\frac{1}{\rho_{\epsilon} \gamma} \mathrm{e}^{\alpha+P(-\operatorname{Re}(s) \tau)} \leqslant \eta<1
$$

Thus we have immediately that $\left|Z_{n}(s)\right| \leqslant C_{\epsilon}^{\prime}|\operatorname{Im}(s)|^{2+\epsilon} \rho_{\epsilon}^{n}$, for $|\operatorname{Im}(s)|$ large enough and $|\operatorname{Re}(s)|$ close to $s_{0}, C_{\epsilon}^{\prime}>0$ being a constant. We deduce that the generalized Fredholm determinant

$$
\zeta(s)=\exp \left(\sum_{n=1}^{+\infty} \frac{1}{n} Z_{n}(s)\right),
$$

which is defined for $\operatorname{Re}(s)>s_{0}$, can be continued analytically to a domain where $\left|\operatorname{Re}(s)-s_{0}\right| \leqslant$ $\varepsilon_{0}$ and $|\operatorname{Im}(s)|>T_{0}$, for some $T_{0}, \varepsilon_{0}>0$. Moreover, we have the estimate

$$
\exp \left(-C_{\epsilon}^{\prime}|\operatorname{Im}(s)|^{2+\epsilon} \log \left(\frac{1}{1-\rho_{\epsilon}}\right)\right) \leqslant|\zeta(s)| \leqslant \exp \left(C_{\epsilon}^{\prime}|\operatorname{Im}(s)|^{2+\epsilon} \log \left(\frac{1}{1-\rho_{\epsilon}}\right)\right) .
$$

It remains to show that $\zeta(s)$ has no poles on the axis $\operatorname{Re}(s)=s_{0}$ other than $s_{0}$. Assume that $\tau$ is a lattice, i.e. there exist $L: K \rightarrow m \mathbb{Z}$ with $m>0$ and a Lipschitz function $f$ on $K$ such that for all $x \in K$,

$$
\tau(x)=L(x)+f(x)-f \circ T(x) .
$$

$4^{e}$ SÉRIE - TOME $38-2005-N^{\circ} 1$ 
Using the projection $\Pi: \Sigma_{A}^{+} \rightarrow K$ we get the cohomological equation

$$
\tau(\Pi \xi)=L(\Pi \xi)+f(\Pi \xi)-f(\Pi \sigma \xi),
$$

for all $\xi \in \mathcal{F}_{\theta}^{+}$. There exists $0<\theta<1$ such that $\tau \circ \Pi, f \circ \Pi$ and therefore $L \circ \Pi$ are Lipschitz on $\Sigma_{A}^{+}$with respect to $d_{\theta}$ (the standard ultrametic on $\Sigma_{A}^{+}$, see [28], p. 12). We will denote by $\mathcal{F}_{\theta}^{+}$ the Banach space of Lipschitz functions on $\Sigma_{A}^{+}$. Applying the Ruelle-Perron-Frobenius theorem to the transfer operator $L_{-s_{0} \tau}: \mathcal{F}_{\theta}^{+} \rightarrow \mathcal{F}_{\theta}^{+}$defined by

$$
L_{-s_{0} \tau}(g)(x)=\sum_{\sigma y=x} \mathrm{e}^{-s_{0} \tau(\Pi y)} g(y)
$$

we denote by $h$ a positive eigenfunction $h \in \mathcal{F}_{\theta}^{+}$such that

$$
L_{-s_{0} \tau}(h)=\mathrm{e}^{P\left(-s_{0} \tau\right)} h=h .
$$

Set for all $p \in \mathbb{Z}, t_{p}=\frac{2 p \pi}{m}, g_{p}=\mathrm{e}^{i t_{p} f \circ \Pi} h$. Using (1), we get for all $p \in \mathbb{Z}$,

$$
L_{-\left(s_{0}+i t_{p}\right) \tau}\left(g_{p}\right)=g_{p},
$$

which implies by the classical results in [28] (see the example p. 85 and Theorem 5.6, p. 84) that $\zeta(s)$ has a lattice of poles on the line $\operatorname{Re}(s)=s_{0}$, located at $s_{p}=s_{0}+i t_{p}, p \in \mathbb{Z}$. This is obviously a contradiction with our previous result on $\zeta(s)$ and therefore $\tau$ is non-lattice. Applying [28] again, we conclude the proof.

\section{Examples of zeta functions related to a quadratic Julia set}

In this section, we give a simple application of Theorem 1.7 to a family of zeta functions related to the celebrated quadratic family. The proof of property (NLI) for these dynamical systems is a good introduction to the (more technical) proof of (NLI) for Fuchsian Schottky groups. Let us consider the quadratic map $f: \mathbb{C} \rightarrow \mathbb{C}$, where $c$ is a real parameter and

$$
f(z)=z^{2}+c, \quad c<-2 .
$$

Set $\beta_{c}=(1+\sqrt{1+4|c|}) / 2$ (the largest fixed point of $f$ ) and $\alpha_{c}=\sqrt{|c|-\beta_{c}}$. Let $I_{1}=$ $\left[-\beta_{c},-\alpha_{c}\right]$ and $I_{2}=\left[\alpha_{c}, \beta_{c}\right]$. The map $f: I_{1} \cup I_{2} \rightarrow \mathbb{R}$ satisfies $f\left(I_{1}\right)=f\left(I_{2}\right)=\left[-\beta_{c}, \beta_{c}\right]$ and thus has the Markov property. If we assume ${ }^{3}$ for simplicity that $|c|$ is large enough such that $\alpha_{c}>\frac{1}{2}$, then $f$ is strictly expanding on $I_{1} \cup I_{2}$.

If we take $\beta_{c}<R_{c}<|c|$, then the inverse image $f^{-1}\left(D\left(0, R_{c}\right)\right)$ of the open (complex) disc $D\left(0, R_{c}\right)$ is an open set with two connected components $D_{1} \supset I_{1}$ and $D_{2} \supset I_{2}$ where $D_{j}=$ $g_{j}\left(D\left(0, R_{c}\right)\right)$ with $g_{j}(z)=(-1)^{j} \sqrt{z-c}, j=1,2$, being the holomorphic inverse branches of $f$. It is easy to check that in addition, each $\overline{g_{j}\left(D\left(0, R_{c}\right)\right)} \subset D\left(0, R_{c}\right)$. If $|z|>\beta_{c}, f^{n}(z)$ tends to infinity as $n \rightarrow+\infty$ and therefore the Julia set of $f$ is

$$
J=\bigcap_{n \geqslant 1} f^{-n}\left(D\left(0, R_{c}\right)\right)=\bigcap_{n \geqslant 1} f^{-n}\left(I_{1} \cup I_{2}\right),
$$

\footnotetext{
${ }^{3}$ It actually works for all $c<-2$, one has to consider iterates of $f$ in order to get the expanding property.
} 
and is a Cantor set on the real line. The Bowen formula [8] shows that the Hausdorff dimension $\delta$ of $J$ is given by the unique $t \in \mathbb{R}$ such that $P(-t \tau)=0$ where $P$ is the topological pressure and $\tau=\log \left|f^{\prime}\right|=\log 2|x|$. Using the standard properties of the pressure, one can see that the Hausdorff dimension $\delta$ decays as $|c| \rightarrow+\infty$ and enjoys the asymptotic

$$
\delta \sim 2 \frac{\log 2}{\log |c|} .
$$

Motivated by the quantum resonance theory of open chaotic systems, it is interesting, following M. Zworski [42], to introduce a zeta function related to the hyperbolic dynamics of $f$ on its Julia set. Let $\mathcal{H}$ be the Hilbert space

$$
\mathcal{H}=\left\{h \text { holomorphic on } D_{1} \cup D_{2}: \iint_{D_{1} \cup D_{2}}|h(z)|^{2} d m(z)<+\infty\right\},
$$

where $m$ is the Lebesgue measure. Given $s \in \mathbb{C}$, consider the transfer operator $\mathcal{L}_{s}: \mathcal{H} \rightarrow \mathcal{H}$ defined by

$$
\mathcal{L}_{s}(h)(z)=\sum_{i=1}^{2} \mathrm{e}^{-s \log \left|f^{\prime}\right| \circ g_{i}(z)} h\left(g_{i}(z)\right),
$$

where $\log \left|f^{\prime}\right|$ denotes an holomorphic extension of $\log (2|x|)$ to $D_{1} \cup D_{2}$. Because $\overline{g_{i}\left(D_{j}\right)} \subset D_{i}$ for all $i, j$, it follows from the arguments of $[16,42]$ that $\mathcal{L}_{s}$ is a trace class operator whose Fredholm determinant $\operatorname{det}\left(I-\mathcal{L}_{s}\right)$ is an entire function denoted by $Z(s)$. A computation of the trace shows that for $\operatorname{Re}(s)$ large,

$$
Z(s)=\exp \left(-\sum_{n=1}^{+\infty} \frac{1}{n} \sum_{f^{n} z=z} \frac{\left|\left(f^{n}\right)^{\prime}(z)\right|^{-s}}{1-\left|\left(f^{n}\right)^{\prime}(z)\right|^{-1}}\right) .
$$

We can now give a

Proof of Theorem 1.5. - Consider the Ruelle zeta function

$$
\zeta(s)=\exp \left(\sum_{n=1}^{+\infty} \frac{1}{n} \sum_{f^{n} z=z}\left|\left(f^{n}\right)^{\prime}(z)\right|^{-s}\right) .
$$

Using the Bowen formula, it is clear following [28] that the series defining $\zeta(s)$ converge absolutely and $\zeta(s)$ is analytic and has no zeros in the half-plane $\{\operatorname{Re}(s)>\delta\}$. Moreover, for all $\operatorname{Re}(s)>\delta$, we have from the formula (2),

$$
Z(s)=\prod_{k=0}^{+\infty} \zeta(s+k)^{-1}=\frac{\psi(s)}{\zeta(s)}
$$

where $\psi(s)=\prod_{k=1}^{+\infty} \zeta(s+k)^{-1}$ is (by direct estimates) convergent and non-vanishing in the halfplane $\{\operatorname{Re}(s)>\delta-1\}$. It is therefore enough to work with $\zeta(s)$ and to show that $\tau=\log \left|f^{\prime}\right|$ has the property (NLI) with respect to $f: J \rightarrow J$, and the result will follow directly from Theorem 1.7. Let $\Sigma^{-}$be the space of left sequences $\{1,2\}^{\mathbb{N}_{-}}$, and consider the sequences

$4^{\mathrm{e}}$ SÉRIE - TOME $38-2005-\mathrm{N}^{\circ} 1$ 
$\xi, \eta \in \Sigma^{-}$defined by $\xi_{n}=2$ for all $n \leqslant 0$, and $\eta_{0}=1, \eta_{n}=2$ for all $n \leqslant-1$. Let $u, v \in I_{1}$ with $u>v$. According to our definition of the temporal distance function, we have

$$
\varphi_{\xi, \eta}(u, v)=\sum_{j=1}^{+\infty}\left(\log \left(2\left|g_{2}^{j} u\right|\right)-\log \left(2\left|g_{2}^{j} v\right|\right)\right)-\sum_{j=0}^{+\infty}\left(\log \left(2\left|g_{2}^{j} \circ g_{1} u\right|\right)-\log \left(2\left|g_{2}^{j} \circ g_{1} v\right|\right)\right) .
$$

Since we have $\left|g_{1}(x)\right|=\left|g_{2}(x)\right|$ for all $x \in I_{1} \cup I_{2}$, we have in fact

$$
\begin{aligned}
\varphi_{\xi, \eta}(u, v)= & \sum_{j=2}^{+\infty}\left(\log \left(2\left|g_{2}^{j} u\right|\right)-\log \left(2\left|g_{2}^{j} v\right|\right)\right) \\
& -\sum_{j=2}^{+\infty}\left(\log \left(2\left|g_{2}^{j-1} \circ g_{1} u\right|\right)-\log \left(2\left|g_{2}^{j-1} \circ g_{1} v\right|\right)\right) .
\end{aligned}
$$

We can now observe that the inverse branch $g_{2}$ is strictly increasing while $g_{1}$ is strictly decreasing. In addition $g_{2}\left(I_{1} \cup I_{2}\right) \subset I_{2}$ where $x \mapsto \tau(x)=\log (2|x|)$ is strictly increasing. It follows that for all $j \geqslant 2$,

$$
\log \left(2\left|g_{2}^{j} u\right|\right)>\log \left(2\left|g_{2}^{j} v\right|\right) \quad \text { and } \quad \log \left(2\left|g_{2}^{j-1} \circ g_{1} u\right|\right)<\log \left(2\left|g_{2}^{j-1} \circ g_{1} v\right|\right),
$$

and thus $\varphi_{\xi, \eta}(u, v)>0$ whenever $u>v$.

Choose $v_{0} \in J \cap I_{1}$. Because of the uniformly contracting properties of the inverse branches, $u \mapsto \varphi_{\xi, \eta}\left(u, v_{0}\right)$ has an holomorphic extension to $D_{1}$ on which it is non-constant by the above remarks. It is now clear that there exists $u_{0} \in J \cap D_{1}$ such that

$$
\frac{\partial \varphi_{\xi, \eta}}{\partial u}\left(u_{0}, v_{0}\right) \neq 0
$$

otherwise $u \mapsto \frac{\partial \varphi_{\xi, \eta}}{\partial u}\left(u, v_{0}\right)$ would be vanishing on a perfect set and $u \mapsto \varphi_{\xi, \eta}\left(u, v_{0}\right)$ would be constant on $D_{1}$ by analyticity. The theorem is proved.

We refer the reader to [42] for many examples of numerical computations of zeros of $Z(s)$ from which the size $\varepsilon$ of the zero-free strip can be numerically estimated.

\section{Zeta functions and dynamics on limit sets}

\subsection{Uniformization by Schottky groups and Bowen-Series map}

We describe here how to apply the preceding results to prove Theorem 1.3. In the following, we will view the hyperbolic plane $\mathbb{H}^{2}$ as the unit disc, endowed with the Poincaré metric of constant curvature -1 defined by

$$
d s^{2}=\frac{4}{\left(1-|z|^{2}\right)^{2}} d z d \bar{z}
$$

The boundary of the hyperbolic plane is $\partial \mathbb{H}^{2}=S^{1}$. We will denote by $\widehat{\mathbb{C}}=\mathbb{C} \cup\{\infty\}$ the Riemann sphere.

Let $C_{1}, \ldots, C_{2 p}, p \geqslant 2$, be $2 p$ circles orthogonal to the boundary $S^{1}$ of $\mathbb{H}^{2}$ (so that each $C_{i} \cap \mathbb{H}^{2}$ is a geodesic). Denote by $\mathcal{D}_{1}, \ldots, \mathcal{D}_{2 p}$ the (Euclidian) open discs whose boundaries are 
respectively $C_{1}, \ldots, C_{2 p}$, and assume that $\mathcal{D}_{i} \cap \mathcal{D}_{j}=\emptyset$ for all $i \neq j$. Let $h_{1}, \ldots, h_{p}$ be orientation preserving isometries of $\mathbb{H}^{2}$ such that for all $i, h_{i}\left(\mathcal{D}_{i}\right)=\widehat{\mathbb{C}} \backslash \overline{\mathcal{D}_{2 p-i+1}}$, and set $h_{2 p-i+1}=h_{i}^{-1}$ for all $1 \leqslant i \leqslant p$. The Fuchsian group generated by $h_{1}, \ldots, h_{p}$ is called a Classical Fuchsian Schottky group. For more general definitions, see [36].

If we add the extra condition, which is assumed in the following, that $\overline{\mathcal{D}_{i}} \cap \overline{\mathcal{D}_{j}}=\emptyset$ for all $i \neq j$, then $\Gamma$ has no parabolic elements and the quotient $M=\Gamma \backslash \mathbb{H}^{2}$ is a convex co-compact Riemann surface. The converse is true (see [10]): any convex co-compact surface is isometric to such a quotient $\Gamma \backslash \mathbb{H}^{2}$, where $\Gamma$ is a classical Fuchsian Schottky group.

The topology of a convex co-compact surface is uniquely determined by its genus $g$ and the number of funnels $f$, and such a surface is homeomorphic to a sphere with $g$ handles and $f$ points removed. The Nielsen region of the surface is the convex hull of trapped geodesics and is a compact surface bounded by $f$ closed geodesics. The funnels are determined uniquely by the length of their geodesic boundary. A funnel with a boundary of length $l$ is isometric to $\mathbb{R}_{\lambda}^{+} \times(\mathbb{R} / l \mathbb{Z})_{t}$ with the metric $d s^{2}=d \lambda^{2}+\cosh ^{2}(\lambda) d t^{2}$.

Every element $g \in \Gamma$ (different from the identity map) is an hyperbolic isometry which means that viewed as an isometry of the Poincaré half-plane, $g=h^{-1} \mathrm{e}^{T_{g}} h$, where $h \in \mathrm{PSL}_{2}(\mathbb{R})$ is an isometry and $T_{g}>0$ is called the magnification factor or the translation length of $g$. Each hyperbolic isometry has two fixed points lying on $S^{1}$, one attracting and one repelling.

The axis $A_{g}$ of an hyperbolic isometry $g$ is the unique geodesic invariant by $g$ and $A_{g}$ is precisely the geodesic joining the two fixed points of $g$. The translation length is an invariant of conjugacy classes and there is a one to one correspondence between the length spectrum of $\Gamma \backslash \mathbb{H}^{2}$ and the translation lengths of conjugacy classes in $\Gamma$.

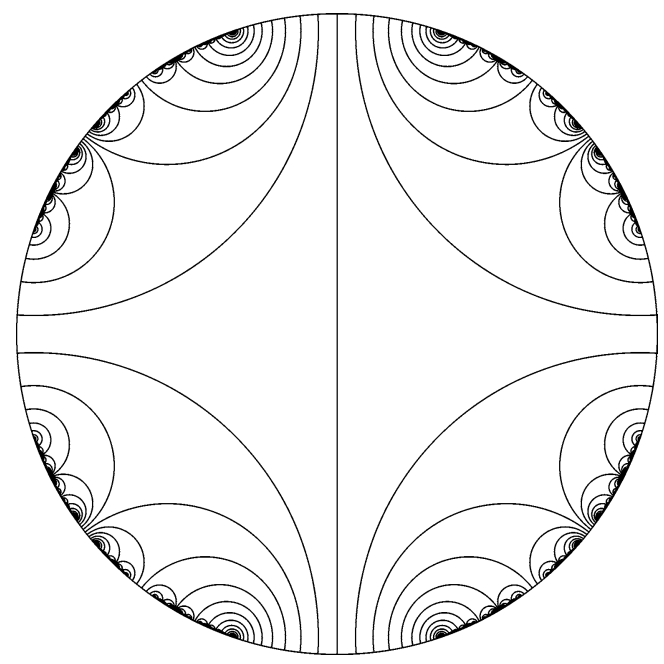

A typical limit set of a Fuchsian Schottky group.

Set $\mathcal{R}=\mathbb{H}^{2} \backslash \bigcup_{i=1}^{2 p} \mathcal{D}_{i}$. The infinite area domain $\mathcal{R}$ is a fundamental domain for the action of $\Gamma$ on $\mathbb{H}^{2}$. Every point of $\mathbb{H}^{2}$ accumulates on the boundary $S^{1}$ under the action of $\Gamma$, and we will denote by $\Lambda$ the limit set of $\Gamma$, i.e. the set of its accumulation points on $S^{1}$. The limit set $\Lambda$ has a structure of Cantor set (it is a perfect and totally disconnected compact set) and its Hausdorff dimension $\delta$ verifies $0<\delta<1$. Moreover, it is exactly the topological entropy of the geodesic flow on the unit tangent bundle of $\Gamma \backslash \mathbb{H}^{2}$ (see Sullivan [44,43]). In addition (see for example [8]) the limit set is of pure dimension which means that if $\mathcal{H}_{\delta}$ denotes the $\delta$-dimensional Hausdorff 
measure, then $0<\mathcal{H}_{\delta}(\Lambda)<+\infty$. For all questions related to Fuchsian groups of the second kind and the dimension of their limit set, we refer to the book [27].

Let $\Gamma$ be a Fuchsian Schottky group with the notations introduced above. To apply the formalism of Section 2.1, we need to build an appropriate dynamical system related to the group $\Gamma$. Set $J_{i}=S^{1} \cap \overline{\mathcal{D}_{i}}$ for $i=1, \ldots, 2 p, J=\bigcup_{i=1}^{2 p} J_{i}$. The boundary map $B: J \rightarrow S^{1}$, also called the Nielsen map or the Bowen-Series map (see [8,9]) is defined by $B(x)=h_{i}(x)$ for $x \in J_{i}$. The cylinder sets of length $n$ generated by $B$ are the sets of the form

$$
C_{i_{1} \cdot i_{2} \ldots . i_{n}}=J_{i_{1}} \cap B^{-1}\left(J_{i_{2}}\right) \cap \cdots \cap B^{-n+1}\left(J_{i_{n}}\right),
$$

where the index $i_{j} \in\{1, \ldots, 2 p\}$ and the word $i_{1} \cdot i_{2} \ldots . i_{n}$ is admissible, i.e. $i_{j+1}+i_{j} \neq 2 p+1$ for all $1 \leqslant j \leqslant n-1$.

In general, the map $B$ with respect to the euclidean metric on $S^{1}$ is not expanding on $J$, but one can prove (see $[8,16,21]$ ) that up to a change of metric it is expanding on a neighborhood of $\Lambda$. More precisely, the following result holds and is enough for our purpose.

PROPOSITION 4.1. - There exist $C>0$ and $D>1$ such that for all $N \geqslant 1$ and for all $z$ in a cylinder set of length $N$,

$$
\left|\left(B^{N}\right)^{\prime}(z)\right| \geqslant C D^{N} .
$$

The map $B$ has a Markov property: for all interval $J_{i}$ and $J_{j}$ such that $\operatorname{Int}\left(B\left(J_{i}\right)\right) \cap J_{j} \neq \emptyset$, we have $J_{j} \subset B\left(J_{i}\right)$. The dynamics of $B$ and hence the action of $\Gamma$ can be described very simply using symbolic dynamics. Let $\Sigma^{+}$denote the set of sequences $\left(x_{n}\right)_{n \geqslant 0} \in\{1, \ldots, 2 p\}^{\mathbb{N}}$ with the transition rule $x_{i}+x_{i+1} \neq 2 p+1$ for all $i \geqslant 0$. Let $p: \Sigma^{+} \rightarrow \Lambda$ be defined by

$$
p(x)=\bigcap_{n=0}^{+\infty} B^{-n}\left(J_{x_{n}}\right) .
$$

Because of the Markov property and the uniformly expanding behaviour of $B$, the projection map $p$ is a homeomorphism between the symbolic space and the limit set, see for example the paper of Bowen [8] for a proof.

The distortion function is by definition $\tau(x)=\log \left|B^{\prime}(x)\right|$ and $\tau$ is analytic on $J$. The Proposition 4.1 implies that there exists $N \geqslant 1$ such that

$$
\tau^{N}(z)=\tau(z)+\tau(B z)+\cdots+\tau\left(B^{N-1} z\right)=\log \left|\left(B^{N}\right)^{\prime}(z)\right|>0
$$

for all $z$ in $B^{-N}(J)$. The distortion function is related to the Hausdorff dimension of the limit set by the remarkable Bowen formula (see [8]): the dimension is the unique real zero of the topological pressure functional $s \mapsto P(-s \tau)$. For algorithms computing the Hausdorff dimension of many conformal dynamical systems including Schottky groups, see [20,24].

The most important feature of the map $B$ for our approach is the following.

Proposition 4.2. - Let $\Gamma$ be a Schottky group as defined above. Then there is a one to one correspondence between the primitive closed geodesics $\gamma$ on $\Gamma \backslash \mathbb{H}^{2}$ of length $l(\gamma)$ and the primitive periodic orbits $x, B x, \ldots, B^{n-1} x$ in $\Lambda$ of $B$ with $l(\gamma)=\tau(x)+\tau(B x)+\cdots+$ $\tau\left(B^{n-1} x\right)$.

For a proof of this kind of correspondence for various Fuchsian groups, see [25,31,32]. This implies (see [28]) that for all $\operatorname{Re}(s)>\delta$, the Selberg zeta function can be written as a convergent 
infinite product

$$
Z_{M}(s)=\prod_{k=0}^{+\infty} \frac{1}{\zeta_{M}(s+k)},
$$

where we have

$$
\zeta_{M}(s)=\exp \left(\sum_{n=1}^{+\infty} \frac{1}{n} \sum_{B^{n} x=x} \mathrm{e}^{-s \tau^{n}(x)}\right),
$$

with $\tau^{n}(x)=\tau(x)+\tau(B x)+\cdots+\tau\left(B^{n-1} x\right)$. In view of the uniformization result of [10], this formula holds for any convex co-compact surface. We point out that in that case $Z_{M}(s)$ can also be viewed as a classical Fredholm determinant (see [16]) related to a trace class operator acting on a well chosen sum of Bergman spaces of holomorphic functions. This point of view has already proved to be useful for the estimates of the growth of the Selberg zeta function. However this is useless for our approach which is based on real analysis and ergodic theory and does not need much regularity.

\subsection{Proof of non-local integrability for Fuchsian Schottky groups}

Let us show how to obtain Theorem 1.3 from Theorem 1.7. Consider

$$
U=\bigcup_{i=1}^{2 p} B^{-1}\left(J_{i}\right)
$$

the union of cylinder sets of length two. Clearly, we have $B(U)=\bigcup_{i=1}^{2 p} J_{i}$. Because $S^{1}$ is not included in $B(U)$, the map $B: U \rightarrow S^{1}$ can be conjugated to an analytic map

$$
T: \bigcup_{i=1}^{k} I_{i} \rightarrow[-\pi, \pi]
$$

where $I_{i}$ denotes a closed interval of $[-\pi, \pi]$ corresponding to a length two cylinder set on $S^{1}$, and $k=2 p(2 p-1)$.

The conjugacy map $\phi$ can be chosen conformal, and $\phi$ maps a connected neighborhood of $B(U)$ to a connected neighborhood of $\bigcup_{i=1}^{k} T\left(I_{i}\right)$, and $\phi$ satisfies $\phi(U)=\bigcup_{i=1}^{k} I_{i}$ and for all $x \in U$,

$$
B(x)=\phi^{-1} \circ T \circ \phi(x) .
$$

Thus the map $T$ inherits the properties of $B$ and it is in the class described above. The transition matrix of $T$ can be written and one can check directly that it is aperiodic since the map $B$ has also this property.

Clearly $T$ generates a Cantor set $K$ of same Hausdorff dimension $\delta$, and $\phi(\Lambda)=K$. In addition, the distortion function $\tau$ of $B$ is cohomologous modulo $\phi$ to the distortion function of $T$ : for all $x \in U$ we have

$$
\tau(x)=\log \left|T^{\prime}(\phi(x))\right|+\log \left|\phi^{\prime}(x)\right|-\log \left|\phi^{\prime}(B x)\right| .
$$

$4^{\mathrm{e}}$ SÉRIE - TOME $38-2005-\mathrm{N}^{\circ} 1$ 
This implies that the Ruelle zeta function $\zeta_{M}(s)$ can be written for all $\operatorname{Re}(s)>\delta$ as

$$
\zeta_{M}(s)=\exp \left(\sum_{n=1}^{+\infty} \frac{1}{n} \sum_{T^{n} x=x} \mathrm{e}^{-s \tilde{\tau}^{n}(x)}\right),
$$

where $\tilde{\tau}=\log \left|T^{\prime}\right|$ is the distortion function of $T$. Therefore, the considerations above show that the zeta function $\zeta_{M}(s)$ is a particular case of zeta function related to the class of Markov maps on Cantor sets described above, and it is a purely dynamical problem. Nevertheless, we will need to keep track of the geometric meaning of $B$ as we will discuss this in the next section.

To prove Theorem 1.3 it remains to prove (NLI) in order to apply the general Theorem 1.7. In the following, we prove that the suspension of the map $T$ by the distortion function is nonintegrable if $T$ comes from a boundary map $B$ defined by a classical Fuchsian Schottky group $\Gamma$ generated by $2 p$ isometries. We use the notations introduced in Section 2.1 and denote by $\tau$ the distortion function of $T$.

First we remark that it is enough to prove the non-integrability for the suspension of $B$ by its distortion function. Indeed, the conjugacy relation $B=\phi^{-1} \circ T \circ \phi$ and the cohomological equation relating $\tau$ and $\tilde{\tau}$ show that the corresponding temporal distance functions are the same modulo the diffeomorphism $\phi$. The existence of a non-vanishing derivative on $K$ is equivalent to the existence of a non-vanishing derivative of the temporal distance function on the limit set $\Lambda$.

Given $u, v \in J_{i}$ and $\xi, \eta \in \Sigma^{+}$with $B\left(J_{\xi_{0}}\right) \cap B\left(J_{\eta_{0}}\right) \supset J_{i}$, the expression of the temporal distance $\varphi_{\xi, \eta}(u, v)$ is, according to the previous section, ${ }^{4}$

$$
\begin{aligned}
\varphi_{\xi, \eta}(u, v)= & \sum_{j=0}^{+\infty} \tau\left(h_{\xi_{j}}^{-1} \circ \cdots \circ h_{\xi_{0}}^{-1} u\right)-\tau\left(h_{\xi_{j}}^{-1} \circ \cdots \circ h_{\xi_{0}}^{-1} v\right) \\
& -\sum_{j=0}^{+\infty}\left(\tau\left(h_{\eta_{j}}^{-1} \circ \cdots \circ h_{\eta_{0}}^{-1} u\right)-\tau\left(h_{\eta_{j}}^{-1} \circ \cdots \circ h_{\eta_{0}}^{-1} v\right)\right),
\end{aligned}
$$

where we recall that $h_{1}, \ldots, h_{2 p}$ are the generators of $\Gamma$. The temporal distance function is analytic on $J_{i} \times J_{i}$. To prove this, remember that $\left.B\right|_{J_{i}}=h_{i}$, and as an isometry of the Poincaré disc we can write

$$
h_{i}(z)=\frac{a_{i} z+b_{i}}{\overline{b_{i}} z+\overline{a_{i}}}
$$

where $a_{i}, b_{i} \in \mathbb{C}$ with $\left|a_{i}\right|^{2}-\left|b_{i}\right|^{2}=1$. This implies that

$$
\left|B^{\prime}(z)\right|=\frac{1}{\left|\overline{b_{i}} z+\overline{a_{i}}\right|^{2}}=\frac{1}{\left(\overline{b_{i}} z+\overline{a_{i}}\right)\left(b_{i} \frac{1}{z}+a_{i}\right)},
$$

for all $z \in J_{i}$. Hence $\left|B^{\prime}(z)\right|$ has a non-vanishing holomorphic continuation to a complex neighborhood of $J_{i}$ for all $1 \leqslant i \leqslant 2 p$. Using a holomorphic determination of the logarithm on each $J_{i}$, we get an analytic extension of $\tau$ to a neighborhood $\Omega$ of $J$. Because the inverse branches $h_{\xi_{j}}^{-1} \circ \cdots \circ h_{\xi_{0}}^{-1}$ are uniformly contracting (Proposition 4.1), there exists a complex neighborhood $\Omega^{\prime}$ of $J_{i}$ such that for all $j \geqslant 0, h_{\xi_{j}}^{-1} \circ \cdots \circ h_{\xi_{0}}^{-1}\left(\Omega^{\prime}\right) \subset \Omega$ and $h_{\eta_{j}}^{-1} \circ \cdots \circ h_{\eta_{0}}^{-1}\left(\Omega^{\prime}\right) \subset \Omega$. Then the

\footnotetext{
${ }^{4}$ Here we use the fact that the transition rules in $\Sigma^{+}$are symmetric, hence any sequence in $\Sigma^{+}$may become a negative sequence by reversing it, therefore we can take $\xi, \eta \in \Sigma^{+}$contrary to the general definition in Section 2 where $\Sigma_{A}^{+}$may not be symmetric.
} 
series defining $\varphi_{\xi, \eta}$ are uniformly convergent on every compact subset of $\Omega^{\prime}$ and $\varphi_{\xi, \eta}$ is indeed analytic on $\Omega^{\prime}$.

The proof of the non-integrability follows from two lemmas.

LEMMA 4.3. - The temporal distance function $\varphi_{\xi, \eta}(u, v)$ is equal to zero for all $i$, all $u, v \in J_{i}$ and all $\xi, \eta \in \Sigma^{+}$with $B\left(J_{\xi_{0}}\right) \cap B\left(J_{\eta_{0}}\right) \supset J_{i}$ if and only if $\tau$ is cohomologous on $\Lambda$ to a function constant on length-two cylinders.

LEMMA 4.4. - For a convex co-compact group $\Gamma$, the distortion function $\tau$ cannot be cohomologous to a locally constant function on $\Lambda$.

Assume that (NLI) does not hold. According to the definition, for all $j \in\{1, \ldots, 2 p\}$, $u, v \in J_{i} \cap \Lambda$ and all $\xi, \eta \in \Sigma^{+}$with $B\left(J_{\xi_{0}}\right) \cap B\left(J_{\eta_{0}}\right) \supset J_{i}$, we have

$$
\frac{\partial \varphi_{\xi, \eta}}{\partial u}(u, v)=0 .
$$

Since $\frac{\partial \varphi_{\xi, \eta}}{\partial u}$ is vanishing on $J_{i} \cap \Lambda \times J_{i} \cap \Lambda$ and $J_{i} \cap \Lambda$ has accumulation points, the holomorphy in $u, v$ implies that

$$
\frac{\partial \varphi_{\xi, \eta}}{\partial u}(u, v)=0
$$

for all $u, v \in J_{i}$. Since $\varphi_{\xi, \eta}(v, v)=0$, we have immediately $\varphi_{\xi, \eta}(u, v)=0$ for all $u, v \in J_{i}$. Applying Lemma 4.3, we get a contradiction with Lemma 4.4.

Proof of Lemma 4.3. - This kind of result dates back to Anosov [3], here we give a proof in our setup which is reminiscent of the Sinai Lemma in Symbolic dynamics. See also Dolgopyat [12] for a symbolic version of our proof. Assume that the temporal distance function vanishes identically i.e. $\varphi_{\xi, \eta}(u, v)=0$ for all $u, v \in J_{i}$, and all $\xi, \eta \in \Sigma^{+}$with $B\left(J_{\xi_{0}}\right) \cap B\left(J_{\eta_{0}}\right) \supset J_{i}$. Let us introduce some simplified notations. Given $\xi \in \Sigma^{+}$, and $j \geqslant 0$, we denote by $h_{\xi}^{-j}$ the inverse branch of $B^{j}$ defined by

$$
h_{\xi}^{-j}=h_{\xi_{j}}^{-1} \circ \cdots \circ h_{\xi_{0}}^{-1} .
$$

We choose $z_{1}, \ldots, z_{2 p}$ such that $z_{i} \in J_{i} \cap \Lambda$ and for all $x \in J$, we define $z(x)$ by $z(x)=z_{i}$ if $x \in J_{i}$. Let $\xi: J \rightarrow \Sigma^{+}$be defined by $\xi(x)=p^{-1}(z(x))$. We recall that $p$ is the natural homeomorphism $p: \Sigma^{+} \rightarrow \Lambda$. Then for all $x \in J$ define $g(x)$ by the absolutely convergent series

$$
g(x)=\sum_{j=0}^{+\infty} \tau\left(h_{\xi(x)}^{-j} x\right)-\tau\left(h_{\xi(x)}^{-j} z(x)\right) .
$$

Notice that if $x \in J_{i}, B\left(J_{i}\right) \supset J_{i}$ and $h_{\xi_{0}(x)}^{-1} x=h_{i}^{-1} x$ is well defined. For $x \in \Lambda$, we have

$$
g(B x)=\sum_{j=0}^{+\infty} \tau\left(h_{\xi(B x)}^{-j} B x\right)-\tau\left(h_{\xi(B x)}^{-j} z(B x)\right) .
$$

Since $\varphi_{\xi, \eta}(B x, z(B x))=0$ for all $\xi, \eta$ with $B\left(J_{\xi_{0}}\right) \cap B\left(J_{\eta_{0}}\right) \supset J_{\xi_{0}(B x)}$, we have in fact

$$
g(B x)=\sum_{j=0}^{+\infty} \tau\left(h_{\hat{\xi}(x)}^{-j} B x\right)-\tau\left(h_{\hat{\xi}(x)}^{-j} z(B x)\right),
$$

$4^{\mathrm{e}}$ SÉRIE - TOME $38-2005-\mathrm{N}^{\circ} 1$ 
where $\hat{\xi}(x)$ denotes the word $\hat{\xi}(x)=\xi_{0}(x) \cdot \xi_{0}(x) \cdot \xi_{1}(x) \ldots$ We remark also that

$$
h_{\hat{\xi}(x)}^{-j}(B x)=h_{\xi_{j-1}(x)}^{-1} \circ \cdots \circ h_{\xi_{0}(x)}^{-1}\left(h_{\xi_{0}(x)}^{-1} B x\right)=h_{\xi(x)}^{-j+1}(x) .
$$

As a consequence, after cancellation, we write

$$
\begin{aligned}
g(x)-g(B x) & =\sum_{j=0}^{+\infty} \tau\left(h_{\xi(x)}^{-j} x\right)-\tau\left(h_{\xi(x)}^{-j+1} x\right)-\sum_{j=0}^{+\infty}\left(\tau\left(h_{\xi(x)}^{-j} z(x)\right)-\tau\left(h_{\hat{\xi}(x)}^{-j} z(B x)\right)\right) \\
& =-\tau(x)-f(x),
\end{aligned}
$$

where $f(x)=\sum_{j=0}^{+\infty} \tau\left(h_{\xi(x)}^{-j} z(x)\right)-\tau\left(h_{\hat{\xi}(x)}^{-j} z(B x)\right)$ depends only on $\left(p^{-1} x\right)_{0}$ and $\left(p^{-1} x\right)_{1}$. We have thus proved that $\tau$ is cohomologous on $\Lambda$ to a locally constant function $f$, moreover $f$ is constant on the cylinders of length two i.e. $f \circ p: \Sigma^{+} \rightarrow \mathbb{R}$ depends only on the first two variables. The converse is obvious.

Proof of Lemma 4.4. - To simplify the notations, we give a proof of this fact only when $\tau$ is cohomologous to a function which is constant on the cylinders of length two. This is enough for our purpose and the proof works exactly in the same way in the general case.

Assume that $\tau$ is cohomologous to a function $f$ constant on every cylinder of the type $C_{i . j}$, where $i . j$ is an admissible word of length two. We will use the two first generators $h_{1}, h_{2}$ of the Schottky group $\Gamma$. Let $x_{1} \in J_{1}$ be the unique repelling fixed point of $h_{1}$. We have $\tau^{2}\left(x_{1}\right)=$ $f^{2}\left(x_{1}\right)=2 f\left(x_{1}\right)$. We also know that $\tau^{2}\left(x_{1}\right)=T_{h_{1}^{2}}=2 T_{h_{1}}$ by the correspondence between closed geodesics and periodic points of $B$, and because $f$ is constant on $C_{1.1}, f(x)=T_{h_{1}}$ for all $x \in C_{1.1}$. Consider $g=h_{2} h_{1}$ and let $y$ be the unique repelling fixed point of $g$. We have $\tau^{2}(y)=$ $T_{g}=f(y)+f(B y)$, where $y \in C_{1.2}$ and $B y \in C_{2.1}$. Because $f$ is constant on $C_{1.2}$ and $C_{2.1}$, we get $f^{2}(x)=T_{g}$ for all $x \in C_{1.2 .1}$. Let $z$ be the unique repelling fixed point of $g h_{1}$. We have again

$$
\tau^{3}(z)=T_{g h_{1}}=f(z)+f^{2}(B z) .
$$

Since $z \in C_{1.1}$ and $B z \in C_{1.2 .1}$, we deduce

$$
T_{g h_{1}}=T_{g}+T_{h_{1}}
$$

Here we use some elementary hyperbolic geometry (see Beardon [6]). The two axes of $g$ and $h_{1}$ cannot be parallel (i.e. with a common point at infinity): this would imply that the repelling fixed points of $g, h_{1}$ or the attracting ones are equal. Therefore two cases can occur.

1. The axes $A_{g}$ and $A_{h_{1}}$ intersect in an angle $0<\theta<\pi$ and according to [6] Theorem 7.38.6, we have

$$
\begin{aligned}
\cosh \left(\frac{1}{2} T_{g h_{1}}\right) & =\cosh \left(\frac{1}{2} T_{g}\right) \cosh \left(\frac{1}{2} T_{h_{1}}\right)+\sinh \left(\frac{1}{2} T_{g}\right) \sinh \left(\frac{1}{2} T_{h_{1}}\right) \cos \theta \\
& <\cosh \frac{1}{2}\left(T_{g}+T_{h_{1}}\right) .
\end{aligned}
$$

2. The axes $A_{g}$ and $A_{h_{1}}$ do not cross. Directed from the repelling fixed point to the attracting one, the two axes $A_{g}$ and $A_{h_{1}}$ clearly have the same orientation. We can therefore apply [6], Theorem 7.38.3 to get

$$
\begin{aligned}
\cosh \left(\frac{1}{2} T_{g h_{1}}\right)= & \cosh \rho\left(A_{g}, A_{h_{1}}\right) \sinh \left(\frac{1}{2} T_{g}\right) \sinh \left(\frac{1}{2} T_{h_{1}}\right) \\
& +\cosh \left(\frac{1}{2} T_{g}\right) \cosh \left(\frac{1}{2} T_{h_{1}}\right)>\cosh \frac{1}{2}\left(T_{g}+T_{h_{1}}\right),
\end{aligned}
$$

ANNALES SCIENTIFIQUES DE L'ÉCOLE NORMALE SUPÉRIEURE 
where $\rho\left(A_{g}, A_{h_{1}}\right)$ is the hyperbolic distance between the two axes. In both cases, we have a contradiction with (3). The proof is complete.

\section{Proof of the main estimate}

\subsection{Reduction to the main estimate}

In this subsection, we recall how the transfer operator estimate of Theorem 2.3 can be reduced to an $L^{2}$ estimate. The proof is taken from [11], we have included it because some of the results and notations used here are needed in the next section. We use the notations of Section 2. The main tools we use here are the Ruelle-Perron-Frobenius Theorem and the quasi-compactness of the transfer operator. In the following, $L(K) \supset C^{1}(I)$ denotes the Banach space of Lipschitz functions on $K$ endowed with its standard norm. We recall that given $f \in L(K), P(f)$ denotes the topological pressure.

THEOREM 5.1 (Ruelle-Perron-Frobenius). - Let $f \in L(K)$ be a real-valued function, and let $\mathcal{L}_{f}$ be the transfer operator defined on $L(K)$ by the usual formula

$$
\mathcal{L}_{f}(g)(x)=\sum_{T y=x} \mathrm{e}^{f(y)} g(y) .
$$

Then the following holds.

- There exists a unique probability measure $\nu_{f}$ on I supported by $K$ such that $\mathcal{L}_{f}^{*}\left(\nu_{f}\right)=$ $\mathrm{e}^{P(f)} \nu_{f}$.

- The transfer operator $\mathcal{L}_{f}$ on $L(K)$ is quasi-compact with spectral radius $\mathrm{e}^{P(f)}$.

- In addition, $\mathrm{e}^{P(f)}$ is a simple eigenvalue and has a unique positive eigenfunction $h_{f} \in$ $L(K)$ with $\nu_{f}\left(h_{f}\right)=1$.

- There are no other eigenvalues of modulus $\mathrm{e}^{P(f)}$.

This result can be derived from [28,7] directly. The $T$-invariant measure $h_{f} \nu_{f}$ is the equilibrium measure $\mu_{f}$ of $f$ on the non-wandering set $K$. If $f$ is in $C^{1}(I)$, then one can check by working directly on $C^{1}(I)^{5}$ that $h_{f} \in C^{1}(I)$ and $\mathcal{L}_{f}: C^{1}(I) \rightarrow C^{1}(I)$ has the same spectral properties. See also [4] for a review of this type of results for more general expanding maps.

The first step of the reduction is to normalize $\mathcal{L}_{s}$ (we recall that $\mathcal{L}_{s}$ is just a simplified notation for $\mathcal{L}_{-s \tau}$ ) with $s=a+i b$ by setting

$$
L_{s}=\mathcal{L}_{\tau_{a}-i b \tau}=\mathrm{e}^{-P(-a \tau)} M_{h_{a}}^{-1} \mathcal{L}_{s} M_{h_{a}},
$$

where $\tau_{a}=-a \tau-P(-a \tau)-\log \left(h_{a} \circ T\right)+\log \left(h_{a}\right), h_{a}$ is the normalized eigenfunction of $\mathcal{L}_{-a \tau}$ related to the maximal eigenvalue $\mathrm{e}^{-P(-a \tau)}$, and $M_{h_{a}}$ denotes the multiplication by $h_{a}$. The operator $L_{a}$ is now Markov for all $a \in \mathbb{R}$, i.e. $L_{a}(1)=1$. From the preceding remarks and standard perturbation theory [28] (the transfer operator $\mathcal{L}_{s}$ depends analytically on $s$, hence the eigenfunction $h_{a}$ and the eigenvalue $\mathrm{e}^{P(-a \tau)}$ depend continuously on $a$ for $a$ close to $s_{0}$ ) we see that to obtain the estimates of Theorem 2.3, it is enough to prove them for the operator $L_{s}$.

In the following it is useful to take a different (but equivalent) norm on $C^{1}(I)$, defined by $\|f\|_{(b)}=\|f\|_{\infty}+\frac{1}{|b|}\left\|f^{\prime}\right\|_{\infty}$ for $|b|>0$. Let us recall some standard a priori estimates.

LEMMA 5.2. - There exist some positive constants $C_{2}, C_{1}$ and $0<\rho_{1}<1$, such that for all $\left|a-s_{0}\right|$ small enough and $|b|$ large enough, we have for all $f \in C^{1}(I)$,

\footnotetext{
${ }^{5}$ For example, by using the technique of Birkhoff cones as in the paper of Liverani [22].

$4^{\mathrm{e}}$ SÉRIE - TOME $38-2005-\mathrm{N}^{\circ} 1$
} 
(i) $\left\|\left(L_{s}^{n} f\right)^{\prime}\right\|_{\infty} \leqslant C_{1}|b|\left\|L_{a}^{n} f\right\|_{\infty}+\rho_{1}^{n}\left\|L_{a}^{n}\left(\left|f^{\prime}\right|\right)\right\|_{\infty}$.

(ii) $\left\|L_{s_{0}}^{n}(f)\right\|_{\infty} \leqslant \int_{K}|f| d \nu_{0}+C_{2} \rho_{1}^{n}\|f\|_{L(K)}$,

where the measure $\nu_{0}$ is the equilibrium measure of $\tau_{s_{0}}$.

We point out that (ii) works actually for all $f \in L(K)$. Notice that because $L_{s_{0}}$ is normalized and $P\left(-s_{0} \tau\right)=0$, Theorem 5.1 applied to $\mathcal{L}_{\tau_{s_{0}}}=L_{s_{0}}$ implies $L_{s_{0}}^{*}\left(\nu_{0}\right)=\nu_{0}$. The estimate (i) is often called the key inequality for complex transfer operators, see [28] for a proof in a symbolic setting. The estimate (ii) comes directly from the quasi-compactness of $L_{s}$ acting on $C^{1}(I)$. From (i) we can see that $\left\|L_{s}^{n}\right\|_{(b)}$ is uniformly bounded for all $n \geqslant 0,\left|a-s_{0}\right|$ small enough and $|b|$ large enough. The main estimate of Theorem 2.3 is a consequence of the following result.

Proposition 5.3. - Under the hypothesis of Theorem 2.3, there exist an integer $N>0$ and $0<\rho<1$ such that for all $\left|a-s_{0}\right|$ small enough and $|b|$ large enough,

$$
\int_{K}\left|L_{s}^{n N} W\right|^{2} d \nu_{0} \leqslant \rho^{n},
$$

for all $W \in C^{1}(I)$ with $\|W\|_{(b)} \leqslant 1$.

Let us show how to recover the statement of Theorem 2.3 from this proposition. We take $s=a+i b$ and $N>0$ as in the previous proposition. Set $n=2\left[\frac{C}{N} \log |b|\right]$ and $\tilde{n}=\left[\frac{C}{N} \log |b|\right]$, with $C$ being a positive constant to be chosen later. For all $s \in \mathbb{C}$ and $f \in C^{1}(I)$ with $\|f\|_{(b)} \leqslant 1$ and $x \in I$, we have

$$
\left|L_{s}^{n N}(f)(x)\right|=\left|L_{s}^{(n-\tilde{n}) N}\left(L_{s}^{\tilde{n} N} f\right)(x)\right| \leqslant L_{a}^{(n-\tilde{n}) N}\left(\left|L_{s}^{\tilde{n} N} f\right|\right)(x) .
$$

In the following, we set $m=(n-\tilde{n}) N$ to simplify the notations. We remark that

$$
\begin{aligned}
L_{a}^{m}\left(\left|L_{s}^{\tilde{n} N} f\right|\right)(x) & =\sum_{T^{m} z=x} \mathrm{e}^{\tau_{a}^{m}(z)}\left|L_{s}^{\tilde{n} N} f\right|(z) \\
& =\sum_{T^{m} z=x} \mathrm{e}^{\frac{1}{2} \tau_{a}^{m}(z)+\frac{1}{2}\left(\tau_{a}^{m}(z)-\tau_{s_{0}}^{m}(z)\right)}\left(\mathrm{e}^{\frac{1}{2} \tau_{s_{0}}^{m}(z)}\left|L_{s}^{\tilde{n} N} f\right|(z)\right) .
\end{aligned}
$$

In addition, we have

$$
\begin{aligned}
\left(\tau_{a}^{m}-\tau_{s_{0}}^{m}\right)(z)= & \left(-a+s_{0}\right) \tau^{m}(z)-P(-a \tau)+P\left(-s_{0} \tau\right) \\
& -\log h_{a}(x)+\log h_{s_{0}}(x)+\log h_{a}(z)-\log h_{s_{0}}(z),
\end{aligned}
$$

hence we roughly have the bound

$$
\left|\left(\tau_{a}^{m}-\tau_{s_{0}}^{m}\right)(z)\right| \leqslant m A\left(a, s_{0}\right),
$$

where $A\left(a, s_{0}\right)=\|\tau\|_{\infty}\left|a-s_{0}\right|+\left|P(-a \tau)-P\left(-s_{0} \tau\right)\right|+2\left\|\log h_{a}-\log h_{s_{0}}\right\|_{\infty}$. Using the Cauchy-Schwartz inequality, we get

$$
\left(L_{a}^{m}\left(\left|L_{s}^{\tilde{n} N} f\right|\right)(x)\right)^{2} \leqslant \mathrm{e}^{m A\left(a, s_{0}\right)} L_{s_{0}}^{m}\left(\left|L_{s}^{\tilde{n} N} f\right|^{2}\right)(x) .
$$

Notice that by perturbation theory, for all $\eta>0$, we know that for all $a$ close enough to $s_{0}$ we have $A\left(a, s_{0}\right) \leqslant \eta$. Using the estimate (ii) from Lemma 5.2, we have

$$
\left\|L_{s}^{n N} f\right\|_{\infty}^{2} \leqslant|b|^{C \eta}\left(\int_{K}\left|L_{s}^{\tilde{n} N} f\right|^{2} d \nu_{0}+C_{3} \rho_{1}^{m}|b|\right) .
$$

ANNALES SCIENTIFIQUES DE L'ÉCOLE NORMALE SUPÉRIEURE 
Applying Proposition 5.3, this yields

$$
\left\|L_{s}^{n N} f\right\|_{\infty}^{2} \leqslant|b|^{C \eta}\left(\frac{C_{4}}{|b|^{\frac{C}{N}|\log \rho|}}+\frac{C_{5}}{|b|^{C\left|\log \rho_{1}\right|-1}}\right),
$$

$C_{4}$ and $C_{5}$ being positive constants. Choosing $C$ such that $C\left|\log \rho_{1}\right|>1$ and then taking $\eta$ close enough to 0 , we get for all $\left|a-s_{0}\right|$ small enough and $|b|$ large,

$$
\left\|L_{s}^{n N} f\right\|_{\infty} \leqslant \frac{1}{|b|^{\beta}}
$$

with $\beta>0$. Applying the estimate (i) from Lemma 5.2, we have in addition,

$$
\frac{1}{|b|}\left\|\left(L_{s}^{n N} f\right)^{\prime}\right\|_{\infty} \leqslant C_{1}\left\|L_{a}^{m}\left(L_{s}^{\tilde{n} N} f\right)\right\|_{\infty}+\rho_{1}^{m}\left\|L_{s}^{N \tilde{n}} f\right\|_{(b)} .
$$

Hence, using similar ideas, we get for all $\left|a-s_{0}\right|$ small and $|b|$ large

$$
\left\|L_{s}^{N n}\right\|_{(b)} \leqslant \frac{1}{|b|^{\beta^{\prime}}}
$$

for some $\beta^{\prime}>0$ and $n=\left[\frac{C}{N} \log |b|\right]$. Given $m \in \mathbb{N}$, we can write $m=d N[C / N \log |b|]+r$, where $d, r \in \mathbb{N}$ and $0 \leqslant r \leqslant N[C / N \log |b|]$. Because the iterations of $L_{s}$ with respect to $\|\cdot\|_{|b|}$ are uniformly bounded by a constant $M>0$, we get

$$
\left\|L_{s}^{m}\right\|_{(b)} \leqslant M\left(\frac{1}{|b|^{\beta^{\prime}}}\right)^{d} \leqslant M|b|^{\beta^{\prime}} \rho_{\beta^{\prime}}^{m},
$$

where $0<\rho_{\beta^{\prime}}<1$. Since the previous estimates are valid for all $\beta^{\prime}>0$, with $\beta^{\prime}$ small, by using the fact that $\|\cdot\|_{C^{1}} \leqslant|b|\|\cdot\|_{(b)}$, we get the conclusions of Theorem 2.3.

\subsection{The key lemma}

The rest of this section is devoted to the proof of Proposition 5.3. The proof follows the main ideas of [11], however we will encounter some additional difficulties due to the disconnected structure of $K$.

Given a constant $A>0$, consider the convex cone $\mathcal{C}_{A}$ of functions in $C^{1}(I)$ defined by

$$
\mathcal{C}_{A}=\left\{f \in C^{1}(I): f>0, \text { and }\left|f^{\prime}(x)\right| \leqslant A f(x), \forall x \in I\right\} .
$$

Notice that for all $f \in \mathcal{C}_{A}$, for all $u, v$ belonging to the same connected component of $I$, we have

$$
\mathrm{e}^{-A|u-v|} \leqslant \frac{f(u)}{f(v)} \leqslant \mathrm{e}^{A|u-v|} .
$$

In this section we will prove the following key result.

LEMMA 5.4. - There exist $N>0, A>1$ and a constant $0<\rho<1$ such that for all $s=a+i b$ with $\left|a-s_{0}\right|$ small and $|b|$ large, there exists a finite set $\left(\mathcal{N}_{s}^{J}\right)_{J \in \mathcal{E}_{s}}$ of bounded operators on $C^{1}(I)$ satisfying the conditions below.

$4^{\mathrm{e}}$ SÉRIE - TOME $38-2005-\mathrm{N}^{\circ} 1$ 
(I) The cone $\mathcal{C}_{A|b|}$ is stable by $\mathcal{N}_{s}^{J}$ for all $J \in \mathcal{E}_{s}$.

(II) For all $H \in \mathcal{C}_{A|b|}$ and all $J \in \mathcal{E}_{s}$,

$$
\int_{K}\left|\mathcal{N}_{s}^{J} H\right|^{2} d \nu_{0} \leqslant \rho \int_{K}|H|^{2} d \nu_{0}
$$

(III) Given $H \in \mathcal{C}_{A|b|}$ and $f \in C^{1}(I)$ such that $|f| \leqslant H$ and $\left|f^{\prime}\right| \leqslant A|b| H$, there exists $J \in \mathcal{E}_{s}$ such that we have

$$
\left|L_{s}^{N} f\right| \leqslant \mathcal{N}_{s}^{J} H \quad \text { and } \quad\left|\left(L_{s}^{N} f\right)^{\prime}\right| \leqslant A|b| \mathcal{N}_{s}^{J} H .
$$

Lemma 5.4 implies Proposition 5.3. Indeed, let $f \in C^{1}(I), f \neq 0$ with $\|f\|_{(b)} \leqslant 1$. Set $H=\|f\|_{(b)} 1$. Clearly, $H \in \mathcal{C}_{A|b|}$ and $|f| \leqslant H,\left|f^{\prime}\right| \leqslant|b|\|f\|_{(b)} \leqslant A|b| H$. By an immediate induction, we get for all $n \geqslant 1,\left|L_{s}^{n N} f\right| \leqslant \mathcal{N}_{s}^{J_{n}} \mathcal{N}_{s}^{J_{n-1}} \ldots \mathcal{N}_{s}^{J_{1}} H$ and $\left|\left(L_{s}^{n N} f\right)^{\prime}\right| \leqslant$ $A|b| \mathcal{N}_{s}^{j_{n}} \mathcal{N}_{s}^{j_{n-1}} \ldots \mathcal{N}_{s}^{j_{1}} H$, for some $J_{1}, \ldots, J_{n} \in \mathcal{E}_{s}$. Hence we have

$$
\int_{K}\left|L_{s}^{n N} f\right|^{2} d \nu_{0} \leqslant \int_{K}\left|\mathcal{N}_{s}^{J_{n}} \mathcal{N}_{s}^{J_{n-1}} \ldots \mathcal{N}_{s}^{J_{1}} H\right|^{2} d \nu_{0} \leqslant \rho^{n} \int_{K}|H|^{2} d \nu_{0} \leqslant \rho^{n},
$$

which is the desired estimate.

Before the construction of the Dolgopyat contraction operators $\mathcal{N}_{s}^{J}$, we need to prove the following.

Proposition 5.5. - Assume that $\tau \in C^{1}(I)$ satisfies the condition (NLI). Then there exist $m^{\prime}, m>0, N_{0} \geqslant 0$ such that for all $N \geqslant N_{0}$, there exist $\alpha_{1}^{N}, \alpha_{2}^{N}$ two inverse branches of $T^{N}$ such that

$$
m^{\prime} \geqslant\left|\frac{d}{d u}\left(\tau^{N} \circ \alpha_{1}^{N}-\tau^{N} \circ \alpha_{2}^{N}\right)(u)\right| \geqslant m
$$

for all $u \in I$.

Proof. - The upper bound is a standard fact in hyperbolic dynamics, see Section 4 of this paper for the proof of similar estimates. There exist $I_{j_{0}}, \xi, \eta \in \Sigma_{A}^{-}$with $T\left(I_{\xi_{0}}\right) \cap T\left(I_{\eta_{0}}\right) \supset I_{j_{0}}$, and $u_{0}, v_{0} \in K \cap I_{j_{0}}$ such that $\frac{\partial \varphi_{\xi, \eta}}{\partial u}\left(u_{0}, v_{0}\right) \neq 0$. For all $u \in I_{j_{0}}$, set $\Phi(u)=\varphi_{\xi, \eta}\left(u, v_{0}\right)$.

Clearly, the non-vanishing derivative implies that there exist $\varepsilon>0$ and an open interval $\mathcal{U}$ with

$$
I_{j_{0}} \supset \mathcal{U} \ni u_{0},
$$

such that $\left|\Phi^{\prime}(u)\right| \geqslant \varepsilon$ for all $u \in \mathcal{U}$. For all $N \geqslant 1$ set $\beta_{1}^{N}=T_{\xi_{-N+1}}^{-1} \circ \cdots \circ T_{\xi_{0}}^{-1}$ and $\beta_{2}^{N}=$ $T_{\eta_{-N+1}}^{-1} \circ \cdots \circ T_{\eta_{0}}^{-1}$. These maps are two inverse branches of $T^{N}$ defined on $I_{j_{0}}$ and by definition of $\Phi$ for all $u \in \mathcal{U}$, we have

$$
\begin{aligned}
\Phi^{\prime}(u)= & \frac{d}{d u}\left(\tau^{N} \circ \beta_{1}^{N}-\tau^{N} \circ \beta_{2}^{N}\right)(u)+\sum_{p \geqslant N} \frac{\tau^{\prime}\left(T_{\xi_{-p}}^{-1} \circ \cdots \circ T_{\xi_{0}}^{-1} u\right)}{T^{\prime}\left(T_{\xi_{-p}}^{-1} \circ \cdots \circ T_{\xi_{0}}^{-1} u\right) \ldots T^{\prime}\left(T_{\xi_{0}}^{-1} u\right)} \\
& -\sum_{p \geqslant N} \frac{\tau^{\prime}\left(T_{\eta_{-p}}^{-1} \circ \cdots \circ T_{\eta_{0}}^{-1} u\right)}{T^{\prime}\left(T_{\eta_{-p}}^{-1} \circ \cdots \circ T_{\eta_{0}}^{-1} u\right) \ldots T^{\prime}\left(T_{\eta_{0}}^{-1} u\right)} .
\end{aligned}
$$

ANNALES SCIENTIFIQUES DE L'ÉCOLE NORMALE SUPÉRIEURE 
Hence for all $u \in \mathcal{U}$, we can write

$$
\varepsilon \leqslant\left|\Phi^{\prime}(u)\right| \leqslant\left|\frac{d}{d u}\left(\tau^{N} \circ \beta_{1}^{N}-\tau^{N} \circ \beta_{2}^{N}\right)(u)\right|+\frac{D}{\gamma^{N}} \frac{2\left\|\tau^{\prime}\right\|_{\infty}}{\gamma-1},
$$

where $\gamma$ is the expanding rate of $T$. As a consequence, for all $N$ large enough (say $N \geqslant N_{1}$ ), we have

$$
\left|\frac{d}{d u}\left(\tau^{N} \circ \beta_{1}^{N}-\tau^{N} \circ \beta_{2}^{N}\right)(u)\right| \geqslant \frac{\varepsilon}{2},
$$

for all $u \in \mathcal{U}$. To complete the proof, set $x=\Pi^{-1}\left(u_{0}\right) \in \Sigma_{A}^{+}$, and set $\varphi^{N_{2}}=T_{x_{0}}^{-1} \circ \cdots \circ T_{x_{N_{2}}}^{-1}$. We have for all $v \in T\left(I_{x_{N_{2}}}\right),\left|u_{0}-\varphi^{N_{2}}(v)\right| \leqslant \frac{D}{\gamma^{N_{2}+1}}$, thus for $N_{2}$ taken large enough, we get $\varphi^{N_{2}}\left(T\left(I_{x_{N_{2}}}\right)\right) \subset \mathcal{U}$. Because of the mixing property of $T$, for all $1 \leqslant j \leqslant k$, there exists ${ }^{6}$ an inverse branch $\varphi_{j}$ of $T^{p_{0}}$ such that $\varphi_{j}\left(I_{j}\right) \subset T\left(I_{x_{N_{2}}}\right)$. Now we set $N=\widetilde{N}+N_{2}+p_{0}$, with $\widetilde{N} \geqslant N_{1}, \alpha_{1}^{N}=\beta_{1}^{\widetilde{N}} \circ \varphi^{N_{2}} \circ \varphi_{j}$ and $\alpha_{2}^{N}=\beta_{2}^{\widetilde{N}} \circ \varphi^{N_{2}} \circ \varphi_{j}$. Therefore we have for all $u \in I_{j}$ and $\tilde{N} \geqslant N_{1}$,

$$
\begin{aligned}
& \left|\frac{d}{d u}\left(\tau^{N} \circ \alpha_{1}^{N}-\tau^{N} \circ \alpha_{2}^{N}\right)(u)\right|=\left|\frac{d}{d u}\left(\tau^{\widetilde{N}} \circ \alpha_{1}^{N}-\tau^{\widetilde{N}} \circ \alpha_{2}^{N}\right)(u)\right| \\
& \quad=\left|\frac{d}{d u}\left(\tau^{\widetilde{N}} \circ \beta_{1}^{\widetilde{N}}-\tau^{\widetilde{N}} \circ \beta_{2}^{\widetilde{N}}\right)\left(\varphi^{N_{2}} \circ \varphi_{j}(u)\right)\right|\left|\left(\varphi^{N_{2}} \circ \varphi_{j}\right)^{\prime}(u)\right| \geqslant \frac{\varepsilon}{2} \frac{1}{\left\|T^{\prime}\right\|_{\infty}^{N_{2}+p_{0}}},
\end{aligned}
$$

and the proof is complete.

In the following, we set $\hat{p}=N_{2}+p_{0}$ and for all $u \in I_{j}, \psi(u)=\varphi^{N_{2}} \circ \varphi_{j}(u)$. Setting $U_{0}=\psi(I)$, by construction we have $U_{0} \subset \mathcal{U}$ and $T^{\hat{p}}: U_{0} \rightarrow I$ is a diffeomorphism whose inverse is $\psi$. The set $U_{0}$ is a disjoint union of $k$ closed intervals, each of them being diffeomorphic to an $I_{j}$ by $\psi$. We will keep the representation $N=\widetilde{N}+\hat{p}$ with $\widetilde{N} \geqslant N_{1}$, such that $\alpha_{i}^{N}=\beta_{i}^{\widetilde{N}} \circ \psi$ are inverse branches of $T^{N}$.

\subsection{The Dolgopyat operators $\mathcal{N}_{s}^{J}$}

As noticed by Stoyanov in [41], the construction of the Dolgopyat operators requires a special partition of $U_{0}$ by arbitrarily small intervals having the so-called "triple intersection property". This additional technical difficulty comes from the fact that the measure $\nu_{0}$ is supported by $K$. In the case of Anosov flows, it is enough to divide $U_{0}$ into closed segments of equal lengths, but in our case we need in addition a partition "well ordered" with respect to the Cantor set.

Proposition 5.6. - There exist some constants $A_{1}^{\prime}, A_{1}>0$ and $A_{2}>0$ such that for all $\varepsilon>0$ small enough, there exists a finite collection $\left(V_{i}\right)_{1 \leqslant i \leqslant q}$ of closed intervals ordered along $U_{0}$ such that:

1. $\mathcal{U} \supset \bigcup_{i=1}^{q} V_{i} \supset U_{0}, V_{i} \cap \operatorname{Int} U_{0} \neq \emptyset$ for all $i$ and $\operatorname{Int} V_{i} \cap \operatorname{Int} V_{j}=\emptyset$ for $i \neq j$.

2. For all $1 \leqslant i \leqslant q$, $\varepsilon A_{1}^{\prime} \leqslant\left|V_{i}\right| \leqslant \varepsilon A_{1}$.

3. For all $1 \leqslant j \leqslant q$, such that $V_{j} \cap K \neq \emptyset$, either $V_{j-1} \cap K \neq \emptyset$ and $V_{j+1} \cap K \neq \emptyset$ or $V_{j-2} \cap K \neq \emptyset$ and $V_{j-1} \cap K \neq \emptyset$ or $V_{j+1} \cap K \neq \emptyset$ and $V_{j+2} \cap K \neq \emptyset$.

4. For all $1 \leqslant i \leqslant q$ such that $V_{i} \cap K \neq \emptyset, V_{i} \cap K \subset U_{0}$ and $\operatorname{dist}\left(\partial V_{i}, K\right) \geqslant A_{2}\left|V_{i}\right|$.

\footnotetext{
${ }^{6}$ We recall to the reader that $p_{0}$ is the least power such that the transition matrix $A^{p_{0}}>0$.

$4^{\mathrm{e}}$ SÉRIE - TOME $38-2005-\mathrm{N}^{\circ} 1$
} 
The third property simply means that intervals with a non-empty intersection with the Cantor set come at least by triads. We will call such a collection a triadic partition of modulus $\varepsilon$. The proof of the existence of triadic partitions of arbitrarily small modulus is given in Section 7.

In the following, we consider a triadic partition $\left(V_{i}\right)_{1 \leqslant i \leqslant q}$ of $U_{0}$ of modulus $\varepsilon=\frac{\varepsilon^{\prime}}{|b|}$ with $\varepsilon^{\prime}$ and $\frac{1}{|b|}$ both small enough. For all $i=1,2$, and $1 \leqslant j \leqslant q$, we set $Z_{j}^{i}=\beta_{i}^{\widetilde{N}}\left(V_{j} \cap U_{0}\right)$. We denote by $X_{j}=\left\{x \in I: \psi(x) \in V_{j}\right\}$, for all $1 \leqslant j \leqslant q$, and we keep in mind that $N=\widetilde{N}+\hat{p}, \widetilde{N}$ being large enough.

For all $j$, the property 4 implies $\operatorname{dist}\left(K \cap V_{j}, \partial V_{j}\right) \geqslant A_{2} A_{1}^{\prime} \frac{\varepsilon^{\prime}}{|b|}$ whenever $K \cap V_{j} \neq \emptyset$. Hence, for all $j$ such that $K \cap V_{j} \neq \emptyset$, there exists a smooth (say $C^{1}$ ) cut off function $\chi_{j}$ on $I$ such that $1 \geqslant \chi_{j} \geqslant 0$ on I, $\chi_{j} \equiv 1$ on the convex hull of $K \cap V_{j}$ and $\chi_{j} \equiv 0$ elsewhere. In addition, we can assume that $\left|\chi_{j}^{\prime}\right| \leqslant A_{3} \frac{|b|}{\varepsilon^{\prime}}$ with a constant $A_{3}$ depending only on the preceding constants. The set $\mathcal{J}_{s}$ is defined by

$$
\mathcal{J}_{s}=\left\{(i, j): i=1,2 \text { and } 1 \leqslant j \leqslant q \text { with } V_{j} \cap K \neq \emptyset\right\} .
$$

In the following we take $0<\theta<1$. Given a non-empty subset $J \subset \mathcal{J}_{s}$, we define a function $\chi_{J} \in C^{1}(I)$ by

$$
\chi_{J}(x)= \begin{cases}1-\theta \chi_{j}\left(\psi\left(T^{N} x\right)\right) & \text { if } x \in Z_{i}^{j} \text { for }(i, j) \in J, \\ 1 & \text { elsewhere. }\end{cases}
$$

The Dolgopyat operator $\mathcal{N}_{s}^{J}$ is simply defined on $C^{1}(I)$ by

$$
\mathcal{N}_{s}^{J}(f)=L_{a}\left(\chi_{J} f\right),
$$

where $s=a+i b$ and $L_{a}$ is the normalized positive transfer operator at $s=a$. We take $A>1$. Let us show that for suitable constants $A, N$ and $\theta$, the cone $\mathcal{C}_{A|b|}$ is stable by the operators $\mathcal{N}_{s}^{J}$. Given $H \in \mathcal{C}_{A|b|}$, for all $x \in I$, we have

$$
\begin{aligned}
\left|\mathcal{N}_{s}^{J}(H)^{\prime}(x)\right| & =\left|L_{a}^{N}\left(\chi_{J} H\right)^{\prime}(x)\right| \\
& \leqslant \sum_{\alpha^{N}} \mathrm{e}^{\tau_{a}^{N}\left(\alpha^{N} x\right)}\left|\left(\tau_{a}^{N} \circ \alpha^{N}\right)^{\prime}(x)\right|\left(\chi_{J} H\right)\left(\alpha^{N} x\right)+\mathrm{e}^{\tau_{a}^{N}\left(\alpha^{N} x\right)}\left|\left(\left(\chi_{J} H\right) \circ \alpha^{N}\right)^{\prime}(x)\right|,
\end{aligned}
$$

where the sum is taken over all inverse branches of $T^{N}$. We have $\left|\left(\chi_{J} \circ \alpha^{N}\right)^{\prime}\right| \leqslant \theta A_{3} \frac{|b|}{\varepsilon^{\prime}}\left\|\psi^{\prime}\right\|_{\infty}$, and we can of course assume that $\left\|\psi^{\prime}\right\|_{\infty} \leqslant 1$ to simplify the further estimates. By a classical bound (see the next section for a proof of a similar result), there exist a constant $\widetilde{C}$ uniform in $N$, and $a$ such that for $a$ close enough to $s_{0},\left|\left(\tau_{a}^{N} \circ \alpha^{N}\right)^{\prime}(x)\right| \leqslant \widetilde{C}$. Therefore we have

$$
\left|\mathcal{N}_{s}^{J}(H)^{\prime}(x)\right| \leqslant \widetilde{C} \mathcal{N}_{s}^{J}(H)(x)+A_{3} \theta \frac{|b|}{\varepsilon^{\prime}} L_{a}^{N}(H)(x)+D A|b| \gamma^{-N} \mathcal{N}_{s}^{J}(H)(x) .
$$

Writing $H=\left(\chi_{J} H\right) / \chi_{J} \leqslant \frac{1}{1-\theta} \chi_{J} H$, we get

$$
\left|\mathcal{N}_{s}^{J}(H)^{\prime}(x)\right| \leqslant\left(\frac{\widetilde{C}}{|b|}+A_{3} \frac{\theta}{(1-\theta) \varepsilon^{\prime}}+D A \gamma^{-N}\right)|b| \mathcal{N}_{s}^{J}(H)(x) \leqslant A|b| \mathcal{N}_{s}^{J}(H)(x),
$$

for all $|b|$ large enough, $a$ close to $s_{0}$ and $\theta, N$ satisfying $\theta \leqslant \min \left(\frac{1}{2}, \varepsilon^{\prime} \frac{A-1}{4 A_{3}}\right)$ and $\gamma^{-N} \leqslant \frac{A-1}{2 D A}$. Let $f \in C^{1}(I)$ and $H \in \mathcal{C}_{A|b|}$ be such that $|f| \leqslant H$ and $\left|f^{\prime}\right| \leqslant A|b| H$. We obtain 


$$
\begin{aligned}
\left|L_{s}^{N}(f)^{\prime}(x)\right| & \leqslant \sum_{\alpha^{N}} \mathrm{e}^{\tau_{a}^{N}\left(\alpha^{N} x\right)}\left|\left(\left(\tau_{a}^{N}+i b \tau^{N}\right) \circ \alpha^{N}\right)^{\prime}(x)\right| H\left(\alpha^{N} x\right)+\mathrm{e}^{\tau_{a}^{N}\left(\alpha^{N} x\right)}\left|\left(f \circ \alpha^{N}\right)^{\prime}(x)\right| \\
& \leqslant D A|b| \gamma^{-N} L_{a}^{N}(H)(x)+\widehat{C}|b| L_{a}^{N}(H)(x),
\end{aligned}
$$

where $\widehat{C}$ is again independent of $N$ and $|b|$ large enough. Using the fact that $\chi_{J}^{-1} \leqslant 2$ if $\theta \leqslant \frac{1}{2}$, we get

$$
\left|L_{s}^{N}(f)^{\prime}(x)\right| \leqslant A|b| \mathcal{N}_{s}^{J}(H)(x),
$$

if $A \geqslant \frac{\widehat{C}}{4}$ and $\gamma^{-N} \leqslant \frac{D}{4}$. In the following, we fix $A \geqslant \max \left(2, \frac{\widehat{C}}{4}\right)$, take $N$ such that $\gamma^{-N} \leqslant \min \left(\frac{A-1}{2 D A}, \frac{D}{4}\right)$, and fix $0<\theta \leqslant \min \left(\frac{1}{2}, \varepsilon^{\prime} \frac{A-1}{4 A_{3}}\right)$.

We need to prove the property (II) i.e. the operators $\mathcal{N}_{s}^{J}$ are contracting the cone in $L^{2}$ norm. For this purpose, and following [11,41], we will say that a subset $J \subset \mathcal{J}_{s}$ is dense if for all $1 \leqslant j \leqslant q$ such that $V_{j} \cap K \neq \emptyset$, there exists $1 \leqslant j^{\prime} \leqslant q$ with $\left(i, j^{\prime}\right) \in J$ for some $i \in\{1,2\}$ such that $\left|j^{\prime}-j\right| \leqslant 2$. Let $J$ be a dense subset, we denote by $W_{J}$ the subset of $K$ defined by

$$
W_{J}=\left\{x \in K: \exists(i, j) \in J: x \in X_{j}\right\} .
$$

The sets $W_{J}$ have the following remarkable property.

Lemma 5.7. - Let $J$ be a dense subset, and $H \in \mathcal{C}_{A|b|}$. Then there exists a constant $\tilde{\varepsilon}>0$ independent of $H,|b|$ and $J$ such that

$$
\int_{W_{J}} H d \nu_{0} \geqslant \tilde{\varepsilon} \int_{K} H d \nu_{0}
$$

The proof is based on the Federer property of the equilibrium measure $\nu_{0}$.

Proposition 5.8. - Let $B(x, r)$ denote the closed ball of center $x$ and radius $r$. Let $\mu$ be an equilibrium measure on $K$ with respect to a Hölder potential $f$. Let $D$ be a real number such that $D>1$. Then there exists $C_{D}>0$ such that for all $x \in K$ and $r>0$,

$$
\mu(B(x, D r)) \leqslant C_{D} \mu(B(x, r)) .
$$

The proof of this Federer property is given in Section 6.

Proof of Lemma 5.7. - We denote by $\mathcal{G}$ the set of indexes in $\{1, \ldots, q\}$ such that $V_{i} \cap K \neq \emptyset$ for all $i \in \mathcal{G}$. We remark that $K \subset \bigcup_{i \in \mathcal{G}} X_{i}$.

Given $i \in \mathcal{G}$, by density of $J$, there exists at least an index $j(i)$ with $\left(i^{\prime}, j(i)\right) \in J$ for some $i^{\prime} \in\{1,2\}$ such that $|j(i)-i| \leqslant 2$. By choosing such a $j(i)$ for all $i \in \mathcal{G}$, we get an application $j: \mathcal{G} \rightarrow \mathcal{G}$. Notice that for all $j^{\prime} \in \mathcal{G}, j^{-1}\left(\left\{j^{\prime}\right\}\right)$ contains at most 5 elements.

For all $i \in \mathcal{G}$, we choose an arbitrary $u_{i} \in K \cap X_{i}$. We have $X_{j(i)} \subset B\left(u_{i}, r\right)$ and $X_{i} \subset$ $B\left(u_{i}, r\right)$, where $B\left(u_{i}, r\right)$ denotes the closed ball of center $u_{i}$ and radius $r=3 A_{1}\left\|\left(\psi^{-1}\right)^{\prime}\right\|_{\infty} \frac{\varepsilon^{\prime}}{|b|}$, simply because $X_{i}=\psi^{-1}\left(V_{i} \cap U_{0}\right)$. Moreover, by property 4 of triadic partitions, we also have $X_{j(i)} \supset B\left(v_{i}, r^{\prime}\right)$, where $r^{\prime}=\frac{1}{2} A_{2} A_{1}^{\prime} \frac{\varepsilon^{\prime}}{|b|} \inf _{U_{0}}\left|\left(\psi^{-1}\right)^{\prime}\right|$, and $v_{i} \in K \cap X_{j(i)}$ such that $\operatorname{dist}\left(v_{i}, \partial X_{j(i)}\right)=\operatorname{dist}\left(K \cap X_{j(i)}, \partial X_{j(i)}\right)$.

Let $H \in \mathcal{C}_{A|b|}$. We have

$$
\int_{K} H d \nu_{0}=\sum_{i \in \mathcal{G}} \int_{X_{i}} H d \nu_{0} \leqslant \sum_{i \in \mathcal{G}_{B\left(u_{i}, r\right)}} \int_{i \in \mathcal{G}} H d \nu_{0} \leqslant \sum_{B\left(u_{i}, r\right)}\left(\max _{i} H\right) \nu_{0}\left(B\left(u_{i}, r\right)\right) .
$$

$4^{\mathrm{e}}$ SÉRIE - TOME $38-2005-\mathrm{N}^{\circ} 1$ 
Moreover, we get also

$$
B\left(v_{i}, 2 r\right) \supset B\left(u_{i}, r\right) \supset B\left(v_{i}, r^{\prime}\right),
$$

hence by the Federer property of the measure $\nu_{0}$ we get

$$
\nu_{0}\left(B\left(u_{i}, r\right)\right) \leqslant \nu_{0}\left(B\left(v_{i}, 2 r\right)\right) \leqslant C_{2 r / r^{\prime}} \nu_{0}\left(B\left(v_{i}, r^{\prime}\right)\right) \leqslant C_{2 r / r^{\prime}} \nu_{0}\left(X_{j(i)}\right) .
$$

Hence for $|b|$ large, we deduce

$$
\begin{aligned}
\int_{K} H d \nu_{0} & \leqslant C^{\prime} \sum_{i \in \mathcal{G}} \mathrm{e}^{2 A|b| r}\left(\min _{X_{j(i)}} H\right) \nu_{0}\left(X_{j(i)}\right) \\
& \leqslant C^{\prime} \mathrm{e}^{C^{\prime \prime}} \sum_{i \in \mathcal{G}} \int_{X_{j(i)}} H d \nu_{0} \\
& \leqslant 5 C^{\prime} \mathrm{e}^{C^{\prime \prime}} \sum_{j: \exists i \text { with }(i, j) \in J} \int_{X_{j}} H d \nu_{0}=5 C^{\prime} \mathrm{e}^{C^{\prime \prime}} \int_{W_{J}} H d \nu_{0},
\end{aligned}
$$

where the constants $C^{\prime}$ and $C^{\prime \prime}$ do not depend on $|b|$. The proof is complete.

We define the set $\mathcal{E}_{s}$ as the set of subsets $J \subset \mathcal{J}_{s}$ such that $J$ is dense. We can prove the following.

Proposition 5.9. - There exists $0<\rho<1$ such that for all $s=a+i b$ with $\left|a-s_{0}\right|$ small and $|b|$ large, for all $H \in \mathcal{C}_{A|b|}$ and for all $J \in \mathcal{E}_{s}$, we have

$$
\int_{K}\left|\mathcal{N}_{s}^{J}(H)\right|^{2} d \nu_{0} \leqslant \rho \int_{K} H^{2} d \nu_{0}
$$

Proof. - Let $H \in \mathcal{C}_{A|b|}$. First, we remark that for all $x \in I$, we have by the Cauchy-Schwarz inequality,

$$
\begin{aligned}
\left(\mathcal{N}_{s}^{J}(H)\right)^{2}(x) & =\left(\sum_{T^{N} y=x} \mathrm{e}^{\tau_{a}^{N}(y)} \chi_{J}(y) H(y)\right)^{2} \\
& \leqslant\left(\sum_{T^{N} y=x} \mathrm{e}^{\tau_{a}^{N}(y)} \chi_{J}^{2}(y)\right)\left(\sum_{T^{N} y=x} \mathrm{e}^{\tau_{a}^{N}(y)} H^{2}(y)\right) \\
& =L_{a}^{N}\left(\chi_{J}^{2}\right)(x) L_{a}^{N}\left(H^{2}\right)(x) \leqslant L_{a}^{N}\left(\chi_{J}\right)(x) L_{a}^{N}\left(H^{2}\right)(x) .
\end{aligned}
$$

For all $x \in W_{J}$ and a well chosen $i \in\{1,2\}$, we have $\chi_{J}\left(\beta_{i}^{\widetilde{N}} \circ \psi(x)\right)=1-\theta$. Notice in addition that if $T^{N} z=x$, then we have roughly $\left|\tau_{a}^{N}(z)\right| \leqslant N B_{a}$, where we can take $B_{a}=$ $a\|\tau\|_{\infty}+|P(-a \tau)|+2\left\|\log h_{a}\right\|_{\infty}$ which is a locally bounded function by perturbation theory. This remark shows that

$$
L_{a}^{N}\left(\chi_{J}\right)(x) \leqslant \sum_{\alpha^{N} \neq \beta_{i}^{\widetilde{N}} \circ \psi} \mathrm{e}^{\tau_{a}^{N} \circ \alpha^{N}(x)}+(1-\theta) \mathrm{e}^{\tau_{a}^{N} \circ \beta_{i}^{\tilde{N}} \circ \psi(x)} \leqslant 1-\theta \mathrm{e}^{-N B_{a}},
$$

ANNALES SCIENTIFIQUES DE L'ÉCOLE NORMALE SUPÉRIEURE 
because $L_{a}^{N}(1)=1$. Writing

$$
\int_{K}\left(\mathcal{N}_{s}^{J}(H)\right)^{2} d \nu_{0}=\int_{W_{J}}\left(\mathcal{N}_{s}^{J}(H)\right)^{2} d \nu_{0}+\int_{K \backslash W_{J}}\left(\mathcal{N}_{s}^{J}(H)\right)^{2} d \nu_{0},
$$

we get by the previous estimate on $W_{J}$ the inequality

$$
\begin{aligned}
\int_{K}\left(\mathcal{N}_{s}^{J}(H)\right)^{2} d \nu_{0} & \leqslant\left(1-\theta \mathrm{e}^{-N B_{a}}\right) \int_{W_{J}} L_{a}^{N}\left(H^{2}\right) d \nu_{0}+\int_{K \backslash W_{J}} L_{a}^{N}\left(H^{2}\right) d \nu_{0} \\
& =\int_{K} L_{a}^{N}\left(H^{2}\right) d \nu_{0}-\theta \mathrm{e}^{-N B_{a}} \int_{W_{J}} L_{a}^{N}\left(H^{2}\right) d \nu_{0} .
\end{aligned}
$$

Now we can apply the preceding lemma to $L_{a}^{N}\left(H^{2}\right)$. Indeed, if we repeat the previous estimates on the stability of the cone $\mathcal{C}_{A|b|}$ by $\mathcal{N}_{s}^{J}$, we find that under the preceding choice of constants, $L_{a}^{N}\left(H^{2}\right) \in \mathcal{C}_{\frac{3}{4} A|b|}$ for all $H \in \mathcal{C}_{A|b|}$. Thus we have

$$
\int_{K}\left(\mathcal{N}_{s}^{J}(H)\right)^{2} d \nu_{0} \leqslant\left(1-\tilde{\varepsilon} \theta \mathrm{e}^{-N B_{a}}\right) \int_{K} L_{a}^{N}\left(H^{2}\right) d \nu_{0} .
$$

Since we have $L_{a}^{N}\left(H^{2}\right) \leqslant \mathrm{e}^{N A\left(a, s_{0}\right)} L_{s_{0}}^{N}\left(H^{2}\right)$, we can use perturbation theory once more to show that there exists $0<\rho<1$ such that for all $a$ close enough to $s_{0}$ we have

$$
\left(1-\tilde{\varepsilon} \theta \mathrm{e}^{-N B_{a}}\right) \mathrm{e}^{N A\left(a, s_{0}\right)} \leqslant \rho<1 .
$$

On the other hand,

$$
\int_{K} L_{s_{0}}^{N}\left(H^{2}\right) d \nu_{0}=\int_{K} H^{2} d \nu_{0}
$$

and the proof is complete.

It remains to show property (III) in order to complete the proof of the key lemma. It is based on the following.

Lemma 5.10. - Let $H \in \mathcal{C}_{A|b|}, f \in C^{1}(I)$ be such that $|f| \leqslant H$ and $\left|f^{\prime}\right| \leqslant A|b| H$. Define the functions $\Theta_{j}: I \rightarrow \mathbb{R}^{+}$for $j=1,2$ by

$$
\begin{aligned}
& \Theta_{1}(x)=\frac{\left|\mathrm{e}^{\left(\tau_{a}^{N}+i b \tau^{N}\right)\left(\alpha_{1}^{N} x\right)} f\left(\alpha_{1}^{N} x\right)+\mathrm{e}^{\left(\tau_{a}^{N}+i b \tau^{N}\right)\left(\alpha_{2}^{N} x\right)} f\left(\alpha_{2}^{N} x\right)\right|}{(1-2 \theta) \mathrm{e}^{\tau_{a}^{N}\left(\alpha_{1}^{N} x\right)} H\left(\alpha_{1}^{N} x\right)+\mathrm{e}^{\tau_{a}^{N}\left(\alpha_{2}^{N} x\right)} H\left(\alpha_{2}^{N} x\right)}, \\
& \Theta_{2}(x)=\frac{\left|\mathrm{e}^{\left(\tau_{a}^{N}+i b \tau^{N}\right)\left(\alpha_{1}^{N} x\right)} f\left(\alpha_{1}^{N} x\right)+\mathrm{e}^{\left(\tau_{a}^{N}+i b \tau^{N}\right)\left(\alpha_{2}^{N} x\right)} f\left(\alpha_{2}^{N} x\right)\right|}{\mathrm{e}^{\tau_{a}^{N}\left(\alpha_{1}^{N} x\right)} H\left(\alpha_{1}^{N} x\right)+(1-2 \theta) \mathrm{e}^{\tau_{a}^{n}\left(\alpha_{2}^{N} x\right)} H\left(\alpha_{2}^{N} x\right)} .
\end{aligned}
$$

Then for $\theta$ and $\varepsilon^{\prime}$ chosen small enough, for all $j$ such that $X_{j} \cap K \neq \emptyset$, there exist $j^{\prime}$ with $\left|j^{\prime}-j\right| \leqslant 2, X_{j^{\prime}} \cap K \neq \emptyset$ and $i \in\{1,2\}$ such that for all $x \in X_{j^{\prime}}$, we have

$$
\Theta_{i}(x) \leqslant 1 .
$$

To prove Lemma 5.10, we will use the following lemmas.

$4^{\mathrm{e}}$ SÉRIE - TOME $38-2005-\mathrm{N}^{\circ} 1$ 
LEMMA 5.11. - Let $Z \subset I$ be an interval with $|Z| \leqslant \frac{c}{|b|}$. Let $H \in \mathcal{C}_{A|b|}$ and $f \in C^{1}(I)$ with $|f| \leqslant H$ and $\left|f^{\prime}\right| \leqslant A|b| H$. Then for $c$ small enough, we have either $|f(u)| \leqslant \frac{3}{4} H(u)$ for all $u \in Z$, or $|f(u)| \geqslant \frac{1}{4} H(u)$ for all $u \in Z$.

Proof. - Assume that there exists $u_{0} \in Z$ such that $\left|f\left(u_{0}\right)\right| \leqslant \frac{1}{4} H\left(u_{0}\right)$. Then for all $u \in Z$ we obtain

$$
\begin{aligned}
|f(u)| & \leqslant\left|f(u)-f\left(u_{0}\right)\right|+\frac{1}{4} H\left(u_{0}\right) \leqslant A|b||Z| \sup _{Z} H+\frac{1}{4} H\left(u_{0}\right) \\
& \leqslant\left(c A+\frac{1}{4}\right) \mathrm{e}^{A c} H(u) \leqslant \frac{3}{4} H(u),
\end{aligned}
$$

for all $c$ small enough.

In the following of the proof, for all $z \in \mathbb{C}, z \neq 0, \arg (z)$ will denote the unique real number $\arg (z) \in(-\pi, \pi]$ such that $|z| \mathrm{e}^{i \arg (z)}=z$.

LEMmA 5.12 (Triangle lemma). - Let $z_{1}, z_{2} \neq 0$ be two complex numbers such that $\left|\frac{z_{1}}{z_{2}}\right| \leqslant L$ and $2 \pi-\varepsilon \geqslant\left|\arg \left(z_{1}\right)-\arg \left(z_{2}\right)\right| \geqslant \varepsilon>0$. Then there exists $0<\delta(L, \varepsilon)<1$ such that

$$
\left|z_{1}+z_{2}\right| \leqslant(1-\delta)\left|z_{1}\right|+\left|z_{2}\right|
$$

Proof. - Apply some elementary trigonometry of euclidean triangles.

Proof of Lemma 5.10. - First, we choose $\varepsilon^{\prime}$ small enough such that the conclusion of Lemma 5.11 is valid for all $Z=Z_{j}^{i}$. It is easy to check that this does not change $A$ and $N$. We assume also that $0<\theta \leqslant \frac{1}{8}$ so that $1-2 \theta \geqslant \frac{3}{4}$. Since $Z_{j}^{i}=\beta_{i}^{\widetilde{N}}\left(V_{j}\right)$, we have $\left|Z_{i}^{j}\right| \leqslant\left(D / \gamma^{\widetilde{N}}\right)\left|V_{j}\right|$, hence it is always possible to assume that $\left|Z_{i}^{j}\right| \leqslant\left|V_{j}\right|$ if $\widetilde{N}$ (and thus $N$ ) is taken large enough.

Let $V_{j}, V_{j+1}, V_{j+2}$ be a triad of intervals such that each of them has a non-empty intersection with the Cantor set. We can assume that $\widehat{X}_{j}=X_{j} \cup X_{j+1} \cup X_{j+2}$ is included in a single connected component of $I$.

Two cases occur. If there exists $j^{\prime} \in\{j, j+1, j+2\}$ such that $|f(u)| \leqslant \frac{3}{4} H(u)$ for all $u \in Z_{j^{\prime}}^{i}$ for some $i \in\{1,2\}$, then clearly $\Theta_{i}(u) \leqslant 1$ for all $u \in Z_{j^{\prime}}^{i}$. If this is not the case, then by Lemma 5.11 we have for all $j^{\prime} \in\{j, j+1, j+2\}$, for all $i \in\{1,2\}$ and for all $u \in Z_{j^{\prime}}^{i}$,

$$
|f(u)| \geqslant \frac{1}{4} H(u)
$$

Our goal is to apply Lemma 5.12 to conclude. For all $x \in \widehat{X_{j}}$, we set

$$
z_{1}(x)=\mathrm{e}^{\left(\tau_{a}^{N}+i b \tau^{N}\right)\left(\alpha_{1}^{N} x\right)} f\left(\alpha_{1}^{N} x\right) ; \quad z_{2}(x)=\mathrm{e}^{\left(\tau_{a}^{N}+i b \tau^{N}\right)\left(\alpha_{2}^{N} x\right)} f\left(\alpha_{2}^{N} x\right) .
$$

We claim that given $j^{\prime} \in\{j, j+1, j+2\}$, we have either $\left|\frac{z_{1}(x)}{z_{2}(x)}\right| \leqslant M$ for all $x \in X_{j^{\prime}}$ or $\left|\frac{z_{2}(x)}{z_{1}(x)}\right| \leqslant M$ for all $x \in X_{j^{\prime}}$, where $M=4 \mathrm{e}^{2 N B_{a}} \mathrm{e}^{2 A \varepsilon^{\prime} A_{1}}$. Indeed, for all $x \in X_{j^{\prime}}$, we can write

$$
\frac{1}{4} \mathrm{e}^{-2 N B_{a}} \frac{H\left(\alpha_{1}^{N} x\right)}{H\left(\alpha_{2}^{N} x\right)} \leqslant\left|\frac{z_{1}(x)}{z_{2}(x)}\right| \leqslant 4 \mathrm{e}^{2 N B_{a}} \frac{H\left(\alpha_{1}^{N} x\right)}{H\left(\alpha_{2}^{N} x\right)} .
$$

ANNALES SCIENTIFIQUES DE L'ÉCOLE NORMALE SUPÉRIEURE 
If there exists $x_{0} \in X_{j^{\prime}}$ such that $\frac{H\left(\alpha_{1}^{N} x_{0}\right)}{H\left(\alpha_{2}^{N} x_{0}\right)} \leqslant 1$, then for all $x \in X_{j^{\prime}}$, one gets

$$
\frac{H\left(\alpha_{1}^{N} x\right)}{H\left(\alpha_{2}^{N} x\right)} \leqslant \frac{\mathrm{e}^{A A_{1} \varepsilon^{\prime}} H\left(\alpha_{1}^{N} x_{0}\right)}{\mathrm{e}^{-A A_{1} \varepsilon^{\prime}} H\left(\alpha_{2}^{N} x_{0}\right)} \leqslant \mathrm{e}^{2 A A_{1} \varepsilon^{\prime}},
$$

and we deduce $\left|\frac{z_{1}(x)}{z_{2}(x)}\right| \leqslant M$. If $\frac{H\left(\alpha_{1}^{N} x\right)}{H\left(\alpha_{2}^{N} x\right)} \geqslant 1$ for all $x \in X_{j^{\prime}}$, then $\left|\frac{z_{2}(x)}{z_{1}(x)}\right| \leqslant 4 \mathrm{e}^{2 N B_{a}} \leqslant M$.

We need in addition to control the relative variations of the arguments of $z_{1}$ and $z_{2}$. Since $\left|z_{i}(x)\right| \geqslant \mathrm{e}^{-N B_{a}} \frac{1}{4} H\left(\alpha_{i}^{N} x\right)>0$ for all $x \in \widehat{X_{j}}$ and $i=1,2$, there exist two $C^{1}$ functions $L_{i}: \widehat{X_{j}} \rightarrow \mathbb{C}$ such that for $i=1,2$ we have $L_{i}^{\prime}(x)=\frac{z_{i}^{\prime}(x)}{z_{i}(x)}$ and $\mathrm{e}^{L_{i}(x)}=z_{i}(x)$ for all $x \in \widehat{X_{j}}$. Indeed, consider for example an arbitrary point $x_{0} \in \widehat{X}_{j}$ and set

$$
L_{i}(x)=\int_{x_{0}}^{x} \frac{z_{i}^{\prime}(t)}{z_{i}(t)} d t+z_{0},
$$

where $z_{0}$ is chosen such that $\mathrm{e}^{z_{0}}=z_{i}\left(x_{0}\right)$. For all $x \in \widehat{X_{j}}$, we set $\Phi(x)=\operatorname{Im}\left(L_{1}(x)\right)-$ $\operatorname{Im}\left(L_{2}(x)\right)$. Taking the derivatives for all $x \in \widehat{X_{j}}$, we get

$$
\begin{aligned}
\Phi^{\prime}(x) & =\operatorname{Im}\left(\frac{z_{1}^{\prime}(x)}{z_{1}(x)}-\frac{z_{2}^{\prime}(x)}{z_{2}(x)}\right) \\
& =b \frac{d}{d x}\left(\tau^{N} \circ \alpha_{1}^{N}-\tau^{N} \circ \alpha_{2}^{N}\right)(x)+\operatorname{Im}\left(\frac{\left(f \circ \alpha_{1}^{N}\right)^{\prime}(x)}{f\left(\alpha_{1}^{N} x\right)}-\frac{\left(f \circ \alpha_{2}^{N}\right)^{\prime}(x)}{f\left(\alpha_{2}^{N} x\right)}\right) .
\end{aligned}
$$

A direct estimate shows that

$$
\left|\frac{\left(f \circ \alpha_{1}^{N}\right)^{\prime}(x)}{f\left(\alpha_{1}^{N} x\right)}-\frac{\left(f \circ \alpha_{2}^{N}\right)^{\prime}(x)}{f\left(\alpha_{2}^{N} x\right)}\right| \leqslant 8 A|b| \frac{D}{\gamma^{N}},
$$

hence using Proposition 5.5, for all $x \in \widehat{X_{j}}$ we have

$$
m-8 A \frac{D}{\gamma^{N}} \leqslant \frac{\left|\Phi^{\prime}(x)\right|}{|b|} \leqslant m^{\prime}+8 A \frac{D}{\gamma^{N}} .
$$

For $x \in X_{j}$ and $x^{\prime} \in X_{j+2}$, we can write by the mean value theorem

$$
\left(m-8 A \frac{D}{\gamma^{N}}\right) A_{1}^{\prime} \varepsilon^{\prime} \inf _{U_{0}}\left|\left(\psi^{-1}\right)^{\prime}\right| \leqslant\left|\Phi(x)-\Phi\left(x^{\prime}\right)\right| \leqslant\left(m^{\prime}+8 A \frac{D}{\gamma^{N}}\right) 3 A_{1} \varepsilon^{\prime}\left\|\left(\psi^{-1}\right)^{\prime}\right\|_{\infty} .
$$

By choosing $N$ large enough, we see that independently of $x, x^{\prime}$, we get

$$
B_{1} \varepsilon^{\prime} \leqslant\left|\Phi(x)-\Phi\left(x^{\prime}\right)\right| \leqslant B_{2} \varepsilon^{\prime}
$$

for some constants $B_{1}, B_{2}>0$, independent of $|b|$. We now adjust $\varepsilon^{\prime}$ such that $\left(B_{2}+\frac{B_{1}}{2}\right) \varepsilon^{\prime} \leqslant \pi$, and we set $\varepsilon=B_{1} \frac{\varepsilon^{\prime}}{4}$. Suppose now that there exist $x \in X_{j}$ and $x^{\prime} \in X_{j+2}$ such that both

$$
\Phi(x), \Phi\left(x^{\prime}\right) \in \bigcup_{k \in \mathbb{Z}}[2 k \pi-\varepsilon, 2 k \pi+\varepsilon] .
$$

$4^{\mathrm{e}}$ SÉRIE - TOME $38-2005-\mathrm{N}^{\circ} 1$ 
Since $\left|\Phi(x)-\Phi\left(x^{\prime}\right)\right| \leqslant B_{2} \varepsilon^{\prime}$, we cannot have

$$
\Phi(x) \in\left[2 k_{1} \pi-\varepsilon, 2 k_{1} \pi+\varepsilon\right] \quad \text { and } \quad \Phi\left(x^{\prime}\right) \in\left[2 k_{2} \pi-\varepsilon, 2 k_{2} \pi+\varepsilon\right],
$$

with $k_{1} \neq k_{2}$. Indeed it would imply that

$$
B_{2} \varepsilon^{\prime} \geqslant\left|\Phi(x)-\Phi\left(x^{\prime}\right)\right| \geqslant 2 \pi-2 \varepsilon=2 \pi-B_{1} \varepsilon^{\prime} / 2,
$$

which is not possible. Therefore we have $B_{1} \varepsilon^{\prime} \leqslant\left|\Phi(x)-\Phi\left(x^{\prime}\right)\right| \leqslant 2 \varepsilon=B_{1} \varepsilon^{\prime} / 2$, which is again a contradiction. As a consequence, there exists $j^{\prime} \in\{j, j+2\}$ such that for all $x \in X_{j^{\prime}}$, $\operatorname{dist}(\Phi(x), 2 \pi \mathbb{Z})>\varepsilon$. Because $\mathrm{e}^{i \Phi(x)}=\mathrm{e}^{i\left(\arg \left(z_{1}\right)-\arg \left(z_{2}\right)\right)}$, the hypotheses of Lemma 5.12 are satisfied. We get either for all $x \in X_{j^{\prime}}$,

$$
\left|z_{1}(x)-z_{2}(x)\right| \leqslant(1-\delta(M, \varepsilon))\left|z_{1}(x)\right|+\left|z_{2}(x)\right|
$$

or for all $x \in X_{j^{\prime}}$,

$$
\left|z_{1}(x)-z_{2}(x)\right| \leqslant(1-\delta(M, \varepsilon))\left|z_{2}(x)\right|+\left|z_{1}(x)\right|,
$$

depending on whether $\left|\frac{z_{1}(x)}{z_{2}(x)}\right| \leqslant M$ or $\left|\frac{z_{2}(x)}{z_{1}(x)}\right| \leqslant M$. By choosing $0<\theta<\frac{1}{2} \delta(M, \varepsilon)$, we have $\Theta_{i}(x) \leqslant 1$ for all $x \in X_{j^{\prime}}$ and some $i \in\{1,2\}$.

Now we are ready to prove the property (III). We assume that the constants $N, A, \varepsilon^{\prime}, \theta$ are chosen so that (I) and (II) are satisfied and Lemma 5.10 holds. Let $f \in C^{1}(I)$ and $H \in \mathcal{C}_{A|b|}$ with $|f| \leqslant H$ and $\left|f^{\prime}\right| \leqslant A|b| H$. We must show that there exists a dense subset $J \in \mathcal{E}_{s}$ such that

$$
\left|L_{s}^{N}(f)\right| \leqslant \mathcal{N}_{s}^{J}(H)
$$

Let $J$ be the set of indexes $(i, j)$ such that $\Theta_{i}(x) \leqslant 1$ for all $x \in X_{j}$. Lemma 5.10 tells us that $J$ is dense. Let $x \in I$. If $x \notin \operatorname{Int} X_{j}$, then for all $y \in I$ such that $T^{N} y=x$, we have $\chi_{J}(y)=1$ because $y \in Z_{j}^{i}$ if and only if $y=\alpha_{i}^{N}(z)$ with $z \in X_{j}$. Consequently, we deduce

$$
\left|L_{s}^{N}(f)(x)\right| \leqslant \sum_{T^{N} y=x} \mathrm{e}^{\tau_{a}^{N}(y)} H(y)=\sum_{T^{N} y=x} \mathrm{e}^{\tau_{a}^{N}(y)} \chi_{J}(y) H(y)=\mathcal{N}_{s}^{J}(H)(x) .
$$

If $x \in \operatorname{Int} X_{j}$, then we apply the following argument.

1. If $(1, j) \in J$ and $(2, j) \notin J$, then for all $y \in I$ with $T^{N} y=x$ different from $\alpha_{1}^{N}(x)$, $\chi_{J}(y)=1$ (different inverse branches of $T^{N}$ have disjoint images). Using the fact that $\Theta_{1}(x) \leqslant 1$, we get

$$
\begin{aligned}
\left|L_{s}^{N}(f)(x)\right| \leqslant & \sum_{\substack{T^{N} y=x \\
y \neq \alpha_{1}^{N} x, \alpha_{2}^{N} x}} \mathrm{e}^{\tau_{a}^{N}(y)} H(y)+(1-2 \theta) \mathrm{e}^{\tau_{a}^{N}\left(\alpha_{1}^{N} x\right)} H\left(\alpha_{1}^{N} x\right) \\
& +\mathrm{e}^{\tau_{a}^{N}\left(\alpha_{2}^{N} x\right)} H\left(\alpha_{2}^{N} x\right) \\
\leqslant & \sum_{T^{N} y=x} \mathrm{e}^{\tau_{a}^{N}(y)} \chi_{J}(y) H(y)=\mathcal{N}_{s}^{J}(H)(x) .
\end{aligned}
$$

The case $(2, j) \in J$ and $(1, j) \notin J$ is symmetric. 
2. If $(1, j) \in J$ and $(2, j) \in J$, then for all $y \in I$ with $T^{N} y=x$ different from $\alpha_{1}^{N}(x), \alpha_{2}^{N}(x)$, we have $\chi_{J}(y)=1$. In addition, $\Theta_{1}(x) \leqslant 1$ and $\Theta_{2}(x) \leqslant 1$. By taking half of the sum of these inequalities we deduce

$$
\begin{aligned}
& \left|\mathrm{e}^{\left(\tau_{a}^{N}+i b \tau^{N}\right)\left(\alpha_{1}^{N} x\right)} f\left(\alpha_{1}^{N} x\right)+\mathrm{e}^{\left(\tau_{a}^{N}+i b \tau^{N}\right)\left(\alpha_{2}^{N} x\right)} f\left(\alpha_{2}^{N} x\right)\right| \\
& \quad \leqslant(1-\theta) \mathrm{e}^{\tau_{a}^{N}\left(\alpha_{1}^{N} x\right)} H\left(\alpha_{1}^{N} x\right)+(1-\theta) \mathrm{e}^{\tau_{a}^{N}\left(\alpha_{2}^{N} x\right)} H\left(\alpha_{2}^{N} x\right) \\
& \quad \leqslant \mathrm{e}^{\tau_{a}^{N}\left(\alpha_{1}^{N} x\right)} \chi_{J}\left(\alpha_{1}^{N} x\right) H\left(\alpha_{1}^{N} x\right)+\mathrm{e}^{\tau_{a}^{N}\left(\alpha_{2}^{N} x\right)} \chi_{J}\left(\alpha_{2}^{N} x\right) H\left(\alpha_{2}^{N} x\right) .
\end{aligned}
$$

This implies that $\left|L_{s}^{N}(f)(x)\right| \leqslant \mathcal{N}_{s}^{J}(H)(x)$.

In view of what we showed at the beginning of this section (formula (4)) Lemma 5.4 is proved.

\section{On the Federer property of equilibrium measures on $K$}

This section is devoted to the proof of the Federer property for an arbitrary $T$-invariant equilibrium measure $\mu_{f}$ on the Cantor set $K$, where the potential $f$ is Hölder on $K$. We assume for simplicity that the topological pressure $P(f)=0$. We recall that $\mu_{f}$ can be defined on any Borel set $A$ by $\mu_{f}(A)=\tilde{\mu}\left(\Pi^{-1} A\right)$, where $\tilde{\mu}$ is the equilibrium state of $f \circ \Pi$ which is a Lipschitz observable on $\Sigma_{A}^{+}$with respect to a well chosen metric $d_{\theta}$.

Because of the ultrametric property of $d_{\theta}$ and the distortion bounds of Gibbs measures, $\tilde{\mu}$ is obviously a Federer measure on $\Sigma_{A}^{+}$. It is also clear that SRB measures for expanding maps of an interval still have this property and more generally for any equilibrium state related to an Hölder potential ${ }^{7}$ on the interval (see the first chapter of N. Anantharaman's Phd thesis [1]).

In our setting this property is non-trivial for the pull-back $\mu_{f}$ with respect to the induced distance on $K$ because it is not (in general) Lipschitz equivalent to $\left(\Sigma_{A}^{+}, d_{\theta}\right)$. However, the quasi-self-similar structure of regular Cantor sets allows us to show the Federer property for an arbitrary equilibrium measure. In the following, $B(x, r)$ denotes the closed ball of center $x$ and radius $r$ on the real line.

Let us recall some notations. If $\alpha=\alpha_{0} \cdot \alpha_{1} \ldots \alpha_{n-1}$ with $\alpha_{i} \in\{1, \ldots, k\}$ is a finite admissible word (i.e. $A\left(\alpha_{i}, \alpha_{i+1}\right)=1$ for all $i$ ), we denote by $C_{\alpha}$ the cylinder set $I_{\alpha_{0}} \cap T^{-1} I_{\alpha_{1}} \cap$ $\cdots \cap T^{-n+1} I_{\alpha}$. Every cylinder set $C_{\alpha}$ is a closed interval. The length of $\alpha$ is by definition $|\alpha|=n$, and the diameter of $C_{\alpha}$ is denoted by $\left|C_{\alpha}\right|$. The main tools we need are the following.

Lemma 6.1. - There exist constants $C>0,0<\delta_{1}<1$ and $0<\delta_{2}<1$ such that for all cylinders $C_{\alpha} \subset C_{\beta}$,

$$
C^{-1} \delta_{1}^{|\alpha|-|\beta|} \leqslant \frac{\left|C_{\alpha}\right|}{\left|C_{\beta}\right|} \leqslant C \delta_{2}^{|\alpha|-|\beta|} .
$$

LEMMA 6.2. - For each cylinder set $C_{\beta}$, we have

$$
C_{\beta} \cap K \subset \bigcup_{C_{\alpha} \subset C_{\beta}} C_{\alpha},
$$

\footnotetext{
${ }^{7}$ If the potential has some discontinuities at the boundary of the Markov partition, then the Federer property could fail Consider for example $T x=2 x \bmod 1$ on $[0,1]$, then the equilibrium state of

$$
\phi(x)= \begin{cases}1 & \text { if } x \in[0,1 / 2) \\ 2 & \text { if } x \in[1 / 2,1]\end{cases}
$$
}

is NOT a Federer measure (work near $1 / 2$ and use the Gibbs estimates).

$4^{\mathrm{e}}$ SÉRIE - TOME $38-2005-\mathrm{N}^{\circ} 1$ 
where $|\alpha|=|\beta|+1$. Moreover there exists a constant $\lambda>0$ such that, given two distinct cylinders $C_{\alpha_{1}}, C_{\alpha_{2}} \subset C_{\beta}$ with $\left|\alpha_{i}\right|=|\beta|+1$ for $i=1,2$, we have

$$
\operatorname{dist}\left(C_{\alpha_{1}}, C_{\alpha_{2}}\right) \geqslant \lambda\left|C_{\beta}\right| \text {. }
$$

Let us show how these two properties imply the Federer property (Proposition 5.8).

Proof of Proposition 5.8. - The basic idea of the proof is to show the existence of two cylinder sets $C_{\alpha}, C_{\beta}$ such that $C_{\alpha} \subset B(x, r), C_{\beta} \supset B(x, D r) \cap K, C_{\alpha} \subset C_{\beta}$, with $|\alpha|-|\beta|$ depending only on $D$.

Indeed, by definition of $\mu$ and the Gibbs property we have

$$
\frac{\mu(B(x, D r))}{\mu(B(x, r))} \leqslant \frac{\mu\left(C_{\beta}\right)}{\mu\left(C_{\alpha}\right)} \leqslant A \mathrm{e}^{f^{|\beta|}(x)-f^{|\alpha|}(x)}
$$

where $x \in \Pi^{-1} C_{\alpha}$, and $A$ is a constant depending only on $f$. Since we can write

$$
f^{|\beta|}(x)-f^{|\alpha|}(x)=-f\left(\sigma^{|\alpha|-1} x\right)-\cdots-f\left(\sigma^{|\beta|} x\right)=-f^{|\alpha|-|\beta|}\left(\sigma^{|\beta|} x\right) \leqslant(|\alpha|-|\beta|)\|f\|_{\infty},
$$

the fact that $|\alpha|-|\beta|$ depends only on $D$ implies that

$$
\frac{\mu(B(x, D r))}{\mu(B(x, r))} \leqslant C_{D}
$$

where $C_{D}=A \mathrm{e}^{(|\alpha|-|\beta|)\|f\|_{\infty}}$ depends also only on $D$.

In the following, our goal is to show the existence of such cylinders in any case. Set for simplicity $J=B(x, r)$ and $J^{\prime}=B(x, D r)$. First, we assume that $J^{\prime} \cap K \subsetneq I_{i}$ for some $i \in\{1, \ldots, k\}$. Let $n=\min \left\{j \geqslant 1: \exists C_{\alpha} \subset J^{\prime},|\alpha|=j\right\}$. Clearly $n$ makes sense and ${ }^{8} n \geqslant 2$.

Let $C_{\alpha} \subset J^{\prime}$ be a cylinder with $|\alpha|=n$. Consider an arbitrary $C_{\alpha^{\prime}} \supset C_{\alpha}$ with $\left|\alpha^{\prime}\right|=n-1$. By definition of $n, C_{\alpha^{\prime}} \not \subset J^{\prime}$, so the following alternative holds.

1. If $C_{\alpha^{\prime}} \supset J^{\prime} \cap K$, by Lemma 6.2 we have

$$
C^{-1} \delta_{1}\left|C_{\alpha^{\prime}}\right| \leqslant\left|C_{\alpha}\right| \leqslant\left|J^{\prime}\right|
$$

2. If $C_{\alpha^{\prime}} \not \supset J^{\prime} \cap K$, there exists a cylinder $C_{\beta^{\prime}}$ with $\left|\beta^{\prime}\right|=n-1$ such that $C_{\alpha^{\prime}}, C_{\beta^{\prime}}$ are consecutive and $J^{\prime} \cap K \subset C_{\alpha^{\prime}} \cup C_{\beta^{\prime}}$. Indeed, $J^{\prime} \cap K$ is covered by a finite union of cylinders related to words of length $n-1$ and none of these cylinders can be included in $J^{\prime}$. Consider now a bigger cylinder $C_{\alpha^{\prime \prime}}$ such that $C_{\alpha^{\prime}} \cup C_{\beta^{\prime}} \subset C_{\alpha^{\prime \prime}}$, and assume that

$$
\left|\alpha^{\prime \prime}\right|=\max \left\{j \geqslant 0: \exists C_{\beta} \supset C_{\alpha^{\prime}} \cup C_{\beta^{\prime}},|\beta|=j\right\} .
$$

The maximality of $\left|\alpha^{\prime \prime}\right|$ implies that $C_{\alpha^{\prime}}$ and $C_{\beta^{\prime}}$ are respectively included in bigger cylinders $C_{\hat{\alpha}^{\prime}}$ and $C_{\hat{\beta}^{\prime}}$ with $\left|\hat{\alpha}^{\prime}\right|=\left|\hat{\beta}^{\prime}\right|=\left|\alpha^{\prime \prime}\right|+1$. Since the "gap" between $C_{\alpha^{\prime}}$ and $C_{\beta^{\prime}}$ is included in $J^{\prime}$, using Lemma 6.2, we deduce that

$$
\left|J^{\prime}\right| \geqslant \operatorname{dist}\left(C_{\alpha^{\prime}}, C_{\beta^{\prime}}\right) \geqslant \operatorname{dist}\left(C_{\hat{\alpha}^{\prime}}, C_{\hat{\beta}^{\prime}}\right) \geqslant \lambda\left|C_{\alpha^{\prime \prime}}\right| .
$$

\footnotetext{
${ }^{8}$ Indeed, since $C_{\alpha} \subset J^{\prime} \subsetneq I_{i}=C_{\alpha_{0}}$, we must have at least $|\alpha| \geqslant 2$.
} 
In both cases we have shown that there exists a cylinder $C_{\beta}$ such that $C_{\beta} \supset J^{\prime} \cap K$ and $\left|C_{\beta}\right| \leqslant C^{\prime}\left|J^{\prime}\right|$, where $C^{\prime}$ does not depend on $r, x$ and $D$.

Finally, there exists a decreasing sequence $\left(C_{\gamma_{i}}\right)_{i \geqslant 0}$ of cylinders such that for all $i$,

$$
x \in C_{\gamma_{i}} \subsetneq \cdots \subsetneq C_{\gamma_{1}} \subsetneq C_{\gamma_{0}} \subsetneq C_{\beta} .
$$

Using Lemma 6.1 and the preceding estimate of $\left|C_{\beta}\right|$, we have

$$
\left|C_{\gamma_{i}}\right| \leqslant C C^{\prime} \delta_{2}^{\left|\gamma_{i}\right|-|\beta|} D r
$$

Thus whenever we have $C C^{\prime} \delta_{2}^{\left|\gamma_{i}\right|-|\beta|} D<1$ i.e. $\left|\gamma_{i}\right|-|\beta|>\frac{\log \left(D C C^{\prime}\right)}{\log \delta_{2}}$, we deduce $C_{\gamma_{i}} \subset J$. Hence there exists a cylinder $C_{\alpha}=C_{\gamma_{i}}$ for some $\left|\gamma_{i}\right|$ large enough such that $C_{\alpha} \subset J$ and $|\alpha|-|\beta|$ depends only on $D$ and the proof is complete. It remains to deal with the case when $J^{\prime} \cap K$ is not included in one of the intervals $I_{i}$. Clearly there exists $I_{i} \ni x$. If $I_{i} \subset J^{\prime}$, then $\left|I_{i}\right| \leqslant\left|J^{\prime}\right|$ and following the same ideas as in the preceding case, one can easily construct $C_{\alpha} \subset J$ and $C_{\alpha} \subset I_{i}$ with $|\alpha|$ depending only on $D$. We complete the proof by noticing that

$$
\frac{\mu\left(J^{\prime}\right)}{\mu(J)} \leqslant \frac{1}{\mu\left(C_{\alpha}\right)} \leqslant C_{D}^{\prime}
$$

where as usual $C_{D}^{\prime}$ depends only on $D$. If $I_{i} \not \subset J^{\prime}$, then by a similar "gap" argument to that previously used, we have

$$
\left|I_{i}\right| \leqslant \frac{\max _{j}\left|I_{j}\right|}{\min _{j \neq l} \operatorname{dist}\left(I_{j}, I_{l}\right)}\left|J^{\prime}\right|,
$$

and we conclude the proof in the same way.

Proof of Lemma 6.1. - Let $x, y \in C_{\alpha}$ be such that $|x-y|=\left|C_{\alpha}\right|$ and let $x^{\prime}, y^{\prime} \in C_{\beta}$ be such that $\left|x^{\prime}-y^{\prime}\right|=\left|C_{\beta}\right|$. Set $n=|\alpha|$ and $m=|\beta|$. We have $\left|T^{n} x-T^{n} y\right|=\left|\left(T^{n}\right)^{\prime}(z)\right|\left|C_{\alpha}\right|$ and $\left|T^{m} x^{\prime}-T^{m} y^{\prime}\right|=\left|\left(T^{m}\right)^{\prime}\left(z^{\prime}\right)\right|\left|C_{\beta}\right|$ for some $z \in C_{\alpha}$ and $z^{\prime} \in C_{\beta}$. The chain rule implies that

$$
\frac{\left|C_{\alpha}\right|}{\left|C_{\beta}\right|}=\frac{\left|T^{n} x-T^{n} y\right|}{\left|T^{m} x^{\prime}-T^{m} y^{\prime}\right|} \times \frac{\left|T^{\prime}\left(T^{m-1} z^{\prime}\right) T^{\prime}\left(T^{m-2} z^{\prime}\right) \ldots T^{\prime}\left(z^{\prime}\right)\right|}{\left|T^{\prime}\left(T^{m-1} z\right) T^{\prime}\left(T^{m-2} z\right) \ldots T^{\prime}(z)\right|} \times \frac{1}{\left|T^{\prime}\left(T^{n-1} z\right) \ldots T^{\prime}\left(T^{m} z\right)\right|} .
$$

In addition, we have $\left|T^{n} x-T^{n} y\right|=\left|T^{n}\left(C_{\alpha}\right)\right|=\left|T\left(I_{\alpha_{n-1}}\right)\right| \geqslant\left|I_{\alpha_{n-1}}\right|$ because $\left.T^{n}\right|_{C_{\alpha}}$ is a diffeomorphism mapping the boundary of $C_{\alpha}$ to the boundary of $T^{n}\left(C_{\alpha}\right)=T\left(I_{\alpha_{n-1}}\right)$ by definition of $C_{\alpha}$. For the same reasons, we can also write

$$
\left|T^{m} x^{\prime}-T^{m} y^{\prime}\right|=\left|T^{m}\left(C_{\beta}\right)\right| \geqslant\left|I_{\beta_{m-1}}\right| .
$$

On the other hand, we have

$$
\frac{\left|T^{\prime}\left(T^{m-1} z^{\prime}\right) T^{\prime}\left(T^{m-2} z^{\prime}\right) \ldots T^{\prime}\left(z^{\prime}\right)\right|}{\left|T^{\prime}\left(T^{m-1} z\right) T^{\prime}\left(T^{m-2} z\right) \ldots T^{\prime}(z)\right|}=\exp \left(\sum_{i=0}^{m-1} \log T^{\prime}\left(T^{i} z\right)-\log T^{\prime}\left(T^{i} z^{\prime}\right)\right) .
$$

Moreover we can write $z=\varphi_{\beta} \hat{z}, z^{\prime}=\varphi_{\beta} \hat{z}^{\prime}$, where $\varphi_{\beta}=T_{\beta_{0}}^{-1} \circ \cdots \circ T_{\beta_{m-1}}^{-1}$ is the local inverse branch of $T^{m}$ related to $C_{\beta}$, and we deduce

$$
\left|\log T^{\prime}\left(T^{i} z\right)-\log T^{\prime}\left(T^{i} z^{\prime}\right)\right| \leqslant\left\|\frac{T^{\prime \prime}}{T^{\prime}}\right\|_{\infty}\left|T^{i} \circ \varphi_{\beta} z-T^{i} \circ \varphi_{\beta} z^{\prime}\right| \leqslant\left\|\frac{T^{\prime \prime}}{T^{\prime}}\right\|_{\infty} \frac{D\left|z-z^{\prime}\right|}{\gamma^{m-i}},
$$

$4^{\mathrm{e}}$ SÉRIE - TOME $38-2005-\mathrm{N}^{\circ} 1$ 
where $\gamma>1$ is the expanding rate of $T$. Consequently,

$$
\left|\sum_{i=0}^{m-1} \log T^{\prime}\left(T^{i} z\right)-\log T^{\prime}\left(T^{i} z^{\prime}\right)\right| \leqslant\left\|\frac{T^{\prime \prime}}{T^{\prime}}\right\|_{\infty} D|I| \frac{1}{\gamma-1} .
$$

Finally, we get the estimates

$$
\exp \left(-\left\|\frac{T^{\prime \prime}}{T^{\prime}}\right\|_{\infty} \frac{D|I|}{\gamma-1}\right) \frac{A^{-1}|I|^{-1}}{\left\|T^{\prime}\right\|_{\infty}^{n-m}} \leqslant \frac{\left|C_{\alpha}\right|}{\left|C_{\beta}\right|} \leqslant \exp \left(\left\|\frac{T^{\prime \prime}}{T^{\prime}}\right\|_{\infty} \frac{D|I|}{\gamma-1}\right) \frac{A D|I|}{\gamma^{n-m}},
$$

where $A=\frac{1}{\min _{1 \leqslant j \leqslant k}\left|I_{j}\right|}$. The proof is complete.

Proof of Lemma 6.2. - The first statement is an obvious consequence of the definition of $K$. Denote by $n$ the length of $\beta$. Let $C_{\alpha}, C_{\alpha^{\prime}}$ be two distinct subcylinders of $C_{\beta}$ with $|\alpha|=\left|\alpha^{\prime}\right|=n+1$. Let $(x, y) \in C_{\alpha} \times C_{\alpha^{\prime}}$ be such that $|x-y|=\operatorname{dist}\left(C_{\alpha}, C_{\alpha^{\prime}}\right)$ and $x^{\prime}, y^{\prime} \in C_{\beta}$ with $\left|x^{\prime}-y^{\prime}\right|=\left|C_{\beta}\right|$. Using a similar argument than that used in the preceding proof, we get

$$
\frac{|x-y|}{\left|x^{\prime}-y^{\prime}\right|}=\frac{\operatorname{dist}\left(C_{\alpha}, C_{\alpha^{\prime}}\right)}{\left|C_{\beta}\right|} \geqslant C^{\prime}\left|T^{n} x-T^{n} y\right|,
$$

where $C^{\prime}$ is a constant depending only on $T$. Since $T^{n} x \in I_{\alpha_{n}}$ and $T^{n} y \in I_{\alpha_{n}^{\prime}}$ with $\alpha_{n} \neq \alpha_{n}^{\prime}$, we have

$$
\left|T^{n} x-T^{n} y\right| \geqslant \min _{i \neq j} \operatorname{dist}\left(I_{i}, I_{j}\right),
$$

independently of $n$, and the proof is complete.

\section{Existence of triadic partitions}

This section is devoted to a proof of the existence of triadic partitions. The ideas are similar to [41], but we have to deal here with a more general family of Cantor sets where the number of subcylinders of a given cylinder may not be constant, in contrast to the case of open billiards in [41]. We use the same notations as in Section 4. The proof is based on the distortion Lemmas 6.1, 6.2 and the following additional results. We recall that $|$.$| denotes the diameter (or the Lebesgue$ measure) when applied to an interval.

LEMmA 7.1. - There exists a constant $B_{1}>0$ such that for all $x \in K$ and all $r>0$, there exists a cylinder set $C_{\alpha}$ such that $C_{\alpha} \subset B(x, r)$ and $\left|C_{\alpha}\right| \geqslant B_{1} r$.

Proof. - Consider $C_{\alpha}$ with $x \in C_{\alpha} \subset B(x, r)$, with $\alpha$ of minimal length. For $x \in K$, such a cylinder clearly exists. Let $C_{\beta} \supset C_{\alpha}$ with $|\beta|=|\alpha|-1$. By minimality of $|\alpha|, C_{\beta} \not \subset B(x, r)$ and thus $\left|C_{\beta}\right| \geqslant r$. By applying Lemma 6.1, we get $\left|C_{\alpha}\right| \geqslant C^{-1} \delta_{1}\left|C_{\beta}\right| \geqslant C^{-1} \delta_{1} r$.

LEMMA 7.2. - Let $C_{\beta}$ be an arbitrary cylinder set. Then there exists a finite set of at least 3 words $\mathcal{A}_{\beta}$ such that

$$
C_{\beta} \cap K \subset \bigcup_{\gamma \in \mathcal{A}_{\beta}} C_{\gamma},
$$

where $C_{\gamma} \subset C_{\beta}$ and $|\gamma|=|\beta|+p_{0}+1$.

ANNALES SCIENTIFIQUES DE L'ÉCOLE NORMALE SUPÉRIEURE 
Proof. - Given a finite admissible word $\alpha$, we denote by $\beta \vee \alpha$ the concatenation of the words $\beta$ and $\alpha$ whenever it makes sense i.e $\beta \vee \alpha$ is admissible. Let $\mathcal{A}_{\beta}$ be the set of admissible words of the type $\beta \vee \alpha$ with $|\alpha|=p_{0}+1$. We have immediately

$$
C_{\beta} \cap K \subset \bigcup_{\gamma \in \mathcal{A}_{\beta}} C_{\gamma},
$$

and by definition, $C_{\gamma} \subset C_{\beta}$ for all $\gamma \in \mathcal{A}_{\beta}$. Set $n+1=|\beta|$ to simplify the notations. Since the number of admissible words of length $p_{0}+2$ starting by $i$ and ending by $j$ is given by the entry $A^{p_{0}+1}(i, j)$ of the matrix $A^{p_{0}+1}$, and because $A^{p_{0}}>0$ (we recall that $A$ is aperiodic), we can write

$$
\begin{aligned}
\sharp \mathcal{A}_{\beta}=\sum_{j=1}^{k} A^{p_{0}+1}\left(\beta_{n}, j\right) & =\sum_{1 \leqslant j, l \leqslant k} A^{p_{0}}\left(\beta_{n}, l\right) A(l, j) \\
& \geqslant \sum_{1 \leqslant j, l \leqslant k} A(l, j) .
\end{aligned}
$$

Using the irreducibility of $A$, we have

$$
\sum_{1 \leqslant j, l \leqslant k} A(l, j) \geqslant k+1
$$

and the proof is complete since $k \geqslant 2$.

Remark. - By Lemma 6.1, we have $B_{2}\left|C_{\beta}\right| \leqslant\left|C_{\gamma}\right| \leqslant B_{3}\left|C_{\beta}\right|$ for all $\gamma \in \mathcal{A}_{\beta}$ and some constants $B_{2}, B_{3}>0$ independent of $\beta, \gamma$. Applying inductively Lemma 6.2, we have for all $\gamma_{1}, \gamma_{2} \in \mathcal{A}_{\beta}$ with $\gamma_{1} \neq \gamma_{2}$, $\operatorname{dist}\left(C_{\gamma_{1}}, C_{\gamma_{2}}\right) \geqslant B_{4}\left|C_{\beta}\right|$, for a suitably chosen constant $B_{4}>0$ independent of $\beta, \gamma$.

Proof of Proposition 5.6. - It is enough to prove the statement for a given connected component of $U_{0}$. Let $U$ denote such a component. Let $\varepsilon>0$ be fixed, $\varepsilon$ being small compared to $|U|$. Set $p=[|U| / \varepsilon]$. We can divide $U$ into $p$ closed intervals $J_{i}$ with disjoint interiors such that $\varepsilon \leqslant\left|J_{i}\right| \leqslant 2 \varepsilon$, and $\bigcup_{i=1}^{p} J_{i}=U$.

For all $1 \leqslant i \leqslant p$, we write $J_{i}=\left[x_{i}, x_{i+1}\right]$. The first step is to move slightly the points $x_{i}$ so that the property 4 is fulfilled. For $2 \leqslant i \leqslant p$, we apply the following choice. If $B\left(x_{i}, \frac{\varepsilon}{8}\right) \cap K=\emptyset$, then we set $\tilde{x}_{i}=x_{i}$. If $B\left(x_{i}, \frac{\varepsilon}{8}\right) \cap K \neq \emptyset$, then we pick $x_{i}^{\prime} \in B\left(x_{i}, \frac{\varepsilon}{8}\right) \cap K$. By Lemma 7.1, there exists a cylinder $C_{\alpha} \subset B\left(x_{i}^{\prime}, \frac{\varepsilon}{8}\right)$ with $\left|C_{\alpha}\right| \geqslant B_{1} \frac{\varepsilon}{8}$. Using Lemma 7.2, there exist at least two consecutive cylinders $C_{\gamma_{1}}, C_{\gamma_{2}}$, included in $C_{\alpha}$, satisfying the estimate $\operatorname{dist}\left(C_{\gamma_{1}}, C_{\gamma_{2}}\right) \geqslant$ $B_{4}\left|C_{\alpha}\right|$. Set $\tilde{x}_{i}=\frac{1}{2}\left(\max C_{\gamma_{1}}+\min C_{\gamma_{2}}\right)$.

In both cases, we have $\left|\tilde{x}_{i}-x_{i}\right| \leqslant \frac{\varepsilon}{4}$ and $\operatorname{dist}\left(\tilde{x}_{i}, K\right) \geqslant \min \left(\frac{\varepsilon}{8}, B_{1} B_{4} \frac{\varepsilon}{16}\right)$. For the boundary points $x_{1}$ and $x_{p+1}$, we can apply the same argument to arrange in addition that $\tilde{x}_{1} \leqslant x_{1}$ and $\tilde{x}_{p+1} \geqslant x_{p+1}$. For all $1 \leqslant i \leqslant p$, set $\widetilde{J}_{i}=\left[\tilde{x}_{i}, \tilde{x}_{i+1}\right]$. For all $1 \leqslant i \leqslant p$, we have $\frac{\varepsilon}{2} \leqslant\left|\widetilde{J}_{i}\right| \leqslant \frac{5}{2} \varepsilon$ and $\operatorname{dist}\left(\partial \widetilde{J}_{i}, K\right) \geqslant B_{5}\left|\widetilde{J}_{i}\right|$ with $B_{5}=\frac{2}{5} \min \left(\frac{B_{1} B_{4}}{16}, \frac{1}{8}\right)$ and $U \subset \bigcup_{i=1}^{p} \widetilde{J}_{i}$, the intervals $\widetilde{J}_{i}$ are still with disjoint interiors. Now this new collection of intervals $\widetilde{J}_{i}$ satisfies the properties 1, 2 and 4. To simplify the notations we will still denote by $J_{i}$ these intervals.

The next step is to apply a subdivision procedure so that the triple intersection property is verified. Consider an interval $J_{i}$ such that $J_{i} \cap K \neq \emptyset$. Taking $x \in J_{i} \cap K \neq \emptyset$, we have $B\left(x, B_{5} \frac{\varepsilon}{2}\right) \subset J_{i}$. Using Lemma 7.1 combined with Lemma 7.2, there exist at least 3 consecutive 
cylinders $C_{\gamma_{1}}, C_{\gamma_{2}}$ and $C_{\gamma_{3}}$ with

$$
C_{\gamma_{1}} \cup C_{\gamma_{2}} \cup C_{\gamma_{3}} \subset B\left(x, B_{5} \frac{\varepsilon}{2}\right) \subset J_{i} .
$$

In addition, we have for $i=1,2, \operatorname{dist}\left(C_{\gamma_{i}}, C_{\gamma_{i+1}}\right) \geqslant B_{4} B_{1} B_{5} \frac{\varepsilon}{2}$. We set

$$
y_{i}=\frac{1}{2}\left(\max C_{\gamma_{1}}+\min C_{\gamma_{2}}\right) \quad \text { and } \quad z_{i}=\frac{1}{2}\left(\max C_{\gamma_{2}}+\min C_{\gamma_{3}}\right) .
$$

Writing $J_{i}^{1}=\left[x_{i}, y_{i}\right], J_{i}^{2}=\left[y_{i}, z_{i}\right], J_{i}^{3}=\left[z_{i}, x_{i+1}\right]$, we have $J_{i}^{j} \cap K \neq \emptyset$ for $j=1,2,3$. Moreover, for all $j=1,2,3$,

$$
B_{1} B_{2} B_{5} \frac{\varepsilon}{2} \leqslant\left|J_{i}^{j}\right| \leqslant \frac{5}{2} \varepsilon \quad \text { and } \quad \operatorname{dist}\left(\partial J_{i}^{j}, K\right) \geqslant \min \left(B_{5} B_{4} B_{1} \frac{\varepsilon}{4}, B_{5} \frac{\varepsilon}{2}\right) .
$$

It is now clear that the set of intervals

$$
\left\{J_{i}: J_{i} \cap K=\emptyset\right\} \cup \bigcup_{j=1}^{3}\left\{J_{i}^{j}: J_{i} \cap K \neq \emptyset\right\}
$$

has all the properties listed in Proposition 5.6.

\section{Acknowledgements}

The author would like to thank Viviane Baladi for helpful comments on an earlier version of this paper. I wish to thank Vesselin Petkov and Maciej Zworski for interesting discussions around the example of the quadratic family. Many thanks are also due to the referee who pointed out numerous misprints and imprecisions. The figure in Section 4 was drawn thanks to a program written by Curtis T. McMullen.

\section{Appendix A}

We prove in this appendix the estimate relating the weighted sum on periodic orbits and transfer operators. In the following we assume that $a=\operatorname{Re}(s)$ is in a compact interval $J$. Let us introduce some additional notations. For all admissible word $\alpha$ of length $n$, $\chi_{\alpha}$ will denote a $C^{1}$ cut-off function such that $\chi_{\alpha} \equiv 1$ on the cylinder set $C_{\alpha}$ and $\chi_{\alpha} \equiv 0$ on the other cylinders of length $n$. Such a cut-off function clearly exists since $|\alpha|=|\beta|$ and $\alpha \neq \beta$ imply that $\operatorname{dist}\left(C_{\alpha}, C_{\beta}\right)>0$.

Given two finite admissible words $\alpha=\alpha_{0} \ldots \alpha_{p}$ and $\beta=\beta_{0} \ldots \beta_{q}$, we denote the concatenation of these words by $\alpha \vee \beta=\alpha_{0} \ldots \alpha_{p} \cdot \beta_{0} \ldots \beta_{q}$, whenever it makes sense i.e. $A\left(\alpha_{p}, \beta_{0}\right)=1$.

For all admissible word $\alpha$ (of length $n$ ), we denote by $x_{\alpha}$ the unique fixed point of $T^{n}$ in $C_{\alpha}$ if it exists, otherwise we choose $x_{\alpha} \in C_{\alpha} \cap K$ such that $x_{\alpha} \notin T\left(I_{\alpha_{n-1}}\right)$. Indeed, if $C_{\alpha} \cap K \subset T\left(I_{\alpha_{n-1}}\right)$, then by the Markov property we have $A\left(\alpha_{n-1}, \alpha_{0}\right)=1$ and $C_{\alpha}$ would contain a periodic point of period $n$ which is $\Pi(\alpha \vee \alpha \vee \cdots)$.

This particular choice of points $x_{\alpha}$ implies that we have

$$
\mathcal{L}_{s}^{n}\left(\chi_{\alpha}\right)\left(x_{\alpha}\right)= \begin{cases}\mathrm{e}^{-s \tau^{n}\left(x_{\alpha}\right)} & \text { if } x_{\alpha} \text { is a periodic point } \\ 0 & \text { otherwise }\end{cases}
$$


Therefore we can write

$$
Z_{n}(s)=\sum_{T^{n} x=x} \mathrm{e}^{-s \tau^{n}(x)}=\sum_{|\alpha|=n} \mathcal{L}_{s}^{n}\left(\chi_{\alpha}\right)\left(x_{\alpha}\right),
$$

and this implies that

$$
\begin{aligned}
\left|Z_{n}(s)-\sum_{i=1}^{k} \mathcal{L}_{s}^{n}\left(\chi_{i}\right)\left(x_{i}\right)\right| & =\left|\sum_{|\alpha|=n} \mathcal{L}_{s}^{n}\left(\chi_{\alpha}\right)\left(x_{\alpha}\right)-\sum_{i=1}^{k} \mathcal{L}_{s}^{n}\left(\chi_{i}\right)\left(x_{i}\right)\right| \\
& \leqslant \sum_{m=2}^{n}\left|\sum_{|\beta|=m} \mathcal{L}_{s}^{n}\left(\chi_{\beta}\right)\left(x_{\beta}\right)-\sum_{|\alpha|=m-1} \mathcal{L}_{s}^{n}\left(\chi_{\alpha}\right)\left(x_{\alpha}\right)\right| .
\end{aligned}
$$

We remark now that for all $x \in K$,

$$
\chi_{\alpha}(x)=\sum_{A\left(\alpha_{m-2}, i\right)=1} \chi_{\alpha \vee i}(x),
$$

hence we get

$$
\begin{aligned}
\sum_{|\alpha|=m-1} \mathcal{L}_{s}^{n}\left(\chi_{\alpha}\right)\left(x_{\alpha}\right) & =\sum_{|\alpha|=m-1} \sum_{A\left(\alpha_{m-2}, i\right)=1} \mathcal{L}_{s}^{n}\left(\chi_{\alpha \vee i}\right)\left(x_{\alpha}\right) \\
& =\sum_{|\beta|=m} \mathcal{L}_{s}^{n}\left(\chi_{\beta}\right)\left(x_{\bar{\beta}}\right)
\end{aligned}
$$

where we have used the notation $\bar{\beta}=\beta_{0} \cdot \beta_{1} \ldots \beta_{m-1}$. Therefore, we get

$$
\begin{aligned}
\left|Z_{n}(s)-\sum_{i=1}^{k} \mathcal{L}_{s}^{n}\left(\chi_{i}\right)\left(x_{i}\right)\right| & \leqslant \sum_{m=2}^{n} \sum_{|\beta|=m}\left\|\mathcal{L}_{s}^{n}\left(\chi_{\beta}\right)\right\|_{C^{1}}\left|x_{\beta}-x_{\bar{\beta}}\right| \\
& \leqslant|I| D \sum_{m=2}^{n} \gamma^{1-m}\left\|\mathcal{L}_{s}^{n-m}\right\|_{C^{1}}\left(\sum_{|\beta|=m}\left\|\mathcal{L}_{s}^{m}\left(\chi_{\beta}\right)\right\|_{C^{1}}\right) .
\end{aligned}
$$

For all $m \geqslant 2$ and all admissible words $\beta$ with $|\beta|=m$, we choose $y_{\beta} \in K$ such that $y_{\beta} \in$ $T\left(I_{\beta_{m-1}}\right)$. For all $x \in I$, we deduce

$$
\mathcal{L}_{s}^{m}\left(\chi_{\beta}\right)(x)= \begin{cases}\mathrm{e}^{-s \tau^{m}\left(T_{\beta}^{-1} x\right)} & \text { if } x \in T\left(I_{\beta_{m-1}}\right), \\ 0 & \text { otherwise }\end{cases}
$$

where $T_{\beta}^{-1}=T_{\beta_{0}}^{-1} \circ \cdots \circ T_{\beta_{m-1}}^{-1}$. For all $x \in I \cap T\left(I_{\beta_{m-1}}\right)$, we have

$$
\left|\mathcal{L}_{s}^{m}\left(\chi_{\beta}\right)(x)\right| \leqslant \mathrm{e}^{a|I| B_{1}} \mathrm{e}^{-a \tau^{m}\left(T_{\beta}^{-1} y_{\beta}\right)}
$$

where $\left|\left(\tau^{m} \circ T_{\beta}^{-1}\right)^{\prime}(x)\right| \leqslant B_{1}$ and $B_{1}$ is a uniform constant (we have already encountered this type of estimates in Section 3 and Section 4). Similarly, for all $x \in I \cap T\left(I_{\beta_{m-1}}\right)$, we have

$$
\left|\left(\mathcal{L}_{s}^{m}\left(\chi_{\beta}\right)\right)^{\prime}(x)\right| \leqslant B_{1}|s| \mathrm{e}^{a|I| B_{1}} \mathrm{e}^{-a \tau^{m}\left(T_{\beta}^{-1} y_{\beta}\right)} .
$$

$4^{\mathrm{e}}$ SÉRIE - TOME $38-2005-\mathrm{N}^{\circ} 1$ 
Since $a$ is in a compact set, there exists a uniform constant $B_{2}$ such that for $|\operatorname{Im}(s)|$ large enough, we have

$$
\sum_{|\beta|=m}\left\|\mathcal{L}_{s}^{m}\left(\chi_{\beta}\right)\right\|_{C^{1}} \leqslant B_{2}|\operatorname{Im}(s)| \sum_{|\beta|=m} \mathrm{e}^{-a \tau^{m}\left(z_{\beta}\right)}
$$

where we set $z_{\beta}=T_{\beta}^{-1} y_{\beta}$. It is well known (see $\left.[28,7]\right)$ that if we denote

$$
\phi_{m}(a)=\left(\sum_{|\beta|=m} \mathrm{e}^{-a \tau^{m}\left(z_{\beta}\right)}\right)^{1 / m},
$$

then we have $\lim _{m \rightarrow+\infty} \phi_{m}(a)=\mathrm{e}^{P(-a \tau)}$. Since the function $a \mapsto \phi_{m}(a)$ is decreasing and converges to a continuous function, a classical theorem of Dini implies that the convergence is in fact uniform on every compact interval. Therefore for all $\varepsilon>0$, there exists $B_{\varepsilon}>0$ such that for all $a \in J$, for all $m \geqslant 0$ and $|\operatorname{Im}(s)|$ large,

$$
\sum_{|\beta|=m}\left\|\mathcal{L}_{s}^{m}\left(\chi_{\beta}\right)\right\|_{C^{1}} \leqslant B_{\varepsilon}|\operatorname{Im}(s)| \mathrm{e}^{m(\varepsilon+P(-a \tau))} .
$$

The proof is complete.

\section{REFERENCES}

[1] Anantharaman N., Géodésiques fermées d'une surface sous contraintes homologiques, Thèse de doctorat, Université Paris 6, 2000.

[2] Anantharaman N., Precise counting results for closed orbits of Anosov flows, Ann. Sci. École Norm. Sup. (4) 33 (1) (2000) 33-56.

[3] Anosov D.V., Geodesic flows on closed Riemannian manifolds of negative curvature, Trudy Mat. Inst. Steklov. 90 (1967) 209.

[4] Baladi V., Positive Transfer Operators and Decay of Correlations, Advanced Series in Nonlinear Dynamics, vol. 16, World Scientific, Singapore, 2000.

[5] BALAdi V., VAlLÉE B., Euclidian algorithms are Gaussian, J. Number Theory (2004), submitted for publication.

[6] BEARdon A.F., The Geometry of Discrete Groups, Graduate Texts in Mathematics, vol. 91, SpringerVerlag, New York, 1995, Corrected reprint of the 1983 original.

[7] BowEN R., Equilibrium States and the Ergodic Theory of Anosov Diffeomorphisms, Lecture Notes in Mathematics, vol. 470, Springer-Verlag, Berlin, 1975.

[8] Bowen R., Hausdorff dimension of quasicircles, Inst. Hautes Études Sci. Publ. Math. 50 (1979) 1125.

[9] Bowen R., Series C., Markov maps associated with Fuchsian groups, Inst. Hautes Études Sci. Publ. Math. 50 (1979) 153-170.

[10] ButTon J., All Fuchsian Schottky groups are classical Schottky groups, in: The Epstein birthday schrift, Geom. Topol. Monogr., vol. 1, Geom. Topol. Publ., Coventry, 1998, pp. 117-125 (electronic).

[11] Dolgopyat D., On decay of correlations in Anosov flows, Ann. of Math. (2) 147 (2) (1998) 357-390.

[12] Dolgopyat D., Prevalence of rapid mixing in hyperbolic flows, Ergodic Theory Dynam. Systems 18 (5) (1998) 1097-1114.

[13] Dolgopyat D., Prevalence of rapid mixing. II. Topological prevalence, Ergodic Theory Dynam. Systems 20 (4) (2000) 1045-1059.

[14] Guillopé L., Zworski M., The wave trace for Riemann surfaces, Geom. Funct. Anal. 9 (6) (1999) $1156-1168$. 
[15] GuILlopé L., Sur la distribution des longueurs des géodésiques fermées d'une surface compacte à bord totalement géodésique, Duke Math. J. 53 (3) (1986) 827-848.

[16] GuILlopé L., LiN K.K., ZWORSKI M., The Selberg zeta function for convex co-compact Schottky groups, Comm. Math. Phys. 245 (1) (2004) 149-176.

[17] Hejhal D.A., The Selberg Trace Formula for PSL(2, $R)$, vol. I, Lecture Notes in Mathematics, vol. 548, Springer-Verlag, Berlin, 1976.

[18] Huber H., Zur analytischen Theorie hyperbolischer Raumformen und Bewegungsgruppen. II, Math. Ann. 142 (1960/1961) 385-398.

[19] IwANIEC H., Spectral Methods of Automorphic Forms, second ed., Graduate Studies in Mathematics, vol. 53, American Mathematical Society, Providence, RI, 2002.

[20] Jenkinson O., Pollicott M., Calculating Hausdorff dimensions of Julia sets and Kleinian limit sets, Amer. J. Math. 124 (3) (2002) 495-545.

[21] LALLEY S.P., Renewal theorems in symbolic dynamics, with applications to geodesic flows, nonEuclidean tessellations and their fractal limits, Acta Math. 163 (1-2) (1989) 1-55.

[22] Liverani C., Decay of correlations, Ann. of Math. (2) 142 (2) (1995) 239-301.

[23] Mazzeo R.R., Melrose R.B., Meromorphic extension of the resolvent on complete spaces with asymptotically constant negative curvature, J. Funct. Anal. 75 (2) (1987) 260-310.

[24] MCMullen C.T., Hausdorff dimension and conformal dynamics. III. Computation of dimension, Amer. J. Math. 120 (4) (1998) 691-721.

[25] Morita T., Markov systems and transfer operators associated with cofinite Fuchsian groups, Ergodic Theory Dynam. Systems 17 (5) (1997) 1147-1181.

[26] NAUd F., Precise asymptotics of the length spectrum for finite geometry Riemann surfaces, IMRN (2004), submitted for publication.

[27] NiChOlLs P.J., The Ergodic Theory of Discrete Groups, London Mathematical Society Lecture Note Series, vol. 143, Cambridge University Press, Cambridge, 1989.

[28] PARry W., Pollicott M., Zeta functions and the periodic orbit structure of hyperbolic dynamics, Astérisque 187-188 (1990) 268.

[29] Patterson S.J., Perry P.A., The divisor of Selberg's zeta function for Kleinian groups, Duke Math. $J .106$ (2) (2001) 321-390. Appendix A by Charles Epstein.

[30] PollicotT M., Error terms in "prime orbit theorems" for locally constant suspended flows, Quart. J. Math. Oxford Ser. (2) 41 (163) (1990) 313-323.

[31] Pollicott M., Some applications of thermodynamic formalism to manifolds with constant negative curvature, Adv. Math. 85 (2) (1991) 161-192.

[32] Pollicott M., Rocha A.C., A remarkable formula for the determinant of the Laplacian, Invent. Math. 130 (2) (1997) 399-414.

[33] Pollicott M., Sharp R., Exponential error terms for growth functions on negatively curved surfaces, Amer. J. Math. 120 (5) (1998) 1019-1042.

[34] Pollicott M., Sharp R., Error terms for closed orbits of hyperbolic flows, Ergodic Theory Dynam. Systems 21 (2) (2001) 545-562.

[35] RANDOL B., On the asymptotic distribution of closed geodesics on compact Riemann surfaces, Trans. Amer. Math. Soc. 233 (1977) 241-247.

[36] Ratcliffe J.G., Foundations of Hyperbolic Manifolds, Graduate Texts in Mathematics, vol. 149, Springer-Verlag, New York, 1994.

[37] Ruelle D., Flots qui ne mélangent pas exponentiellement, C. R. Acad. Sci. Paris Sér. I Math. 296 (4) (1983) 191-193.

[38] RuElle D., An extension of the theory of Fredholm determinants, Inst. Hautes Études Sci. Publ. Math. 72 (1991) 175-193, 1990.

[39] SarnaK P., Prime geodesic theorems, PhD thesis, Stanford University, 1980.

[40] Selberg A., Harmonic analysis and discontinuous groups in weakly symmetric Riemannian spaces with applications to Dirichlet series, J. Indian Math. Soc. (N.S.) 20 (1956) 47-87.

[41] STOYANOV L., Spectrum of the Ruelle operator and exponential decay of correlations for open billiard flows, Amer. J. Math. 123 (4) (2001) 715-759.

[42] STRAIN J., ZWORSKI M., Growth of the zeta functions for a quadratic map and the dimension of the julia set, Nonlinearity 17 (5) (2004) 1607-1622.

$4^{e}$ SÉRIE - TOME $38-2005-\mathrm{N}^{\circ} 1$ 
[43] Sullivan D., The density at infinity of a discrete group of hyperbolic motions, Inst. Hautes Études Sci. Publ. Math. 50 (1979) 171-202.

[44] Sullivan D., Entropy, Hausdorff measures old and new, and limit sets of geometrically finite Kleinian groups, Acta Math. 153 (3-4) (1984) 259-277.

[45] WaLters P., An Introduction to Ergodic Theory, Graduate Texts in Mathematics, vol. 79, SpringerVerlag, New York, 1982.

(Manuscrit reçu le 24 mars 2004 ;

accepté, après révision, le 29 novembre 2004.)

Frédéric NAUD

Department of Mathematics,

University of California Berkeley,

Berkeley, CA 94720-3840, USA

E-mail: fnaud@math.berkeley.edu 